\title{
Numerical Modeling of the Non-Isothermal Positive Column of an $\mathrm{Ar}^{+}$-Laser
}

\author{
1. Zech (a), T. Ertl (a), H. Herold (a), H. Ruder (a) \\ (a) Institute for Theoretical Astrophysics, University of Tübingen
}

W. E. KöHLER (b), W. TiemanN (b)

(b) Siemens Research Laboratories, Plasma Physics, Erlangen

\begin{abstract}
A hydrodynamic description of the positive column is used to study the radial variation of particle densities, drift velocities, temperatures and heat fluxes of electrons, singly-charged ions and neutral atoms and the radial electric field. Elastic collisions between the plasma particles and neutrals as well as Coulomb collisions between ions and electrons are taken into account. The relevant equations to solve are the balance equations of particle densities, momentum, energy and the equations for the heat fluxes for each of the three studied particle types; the Poisson equation has to be added for closure. They form a system of 13 nonlinear differential equations with critical points. One singularity occurs when the ions reach the ion sound velocity which is the case inside the positive column. Therefore, a numerical method for multipoint boundary value problems was used which can also successfully handle removable singular points. The applied relaxation method is an iterative method which demands some preliminary knowledge of the solution looked for. The necessary knowledge can be retrieved from the quasineutral model and from a simplified two-fluid model.
\end{abstract}

\section{Introduction}

For our numerical modelling we choose as an interesting plasma physical example the low pressure discharge of an argon ion laser, which is widely used for applications in medical technics. The laser levels of the $\mathrm{Ar}^{+}$-ion are populated via electron collisions in a cylindrical capillary dc-discharge with a diameter of some $\mathrm{mm}$ and at pressures $\leq 1$ torr, such that the ion mean free path is comparable with the diameter of the positive column.

For a long time two limiting theories have been existing for the positive column of a dc-discharge, the free fall theory of TONKS and LANGMUIR [1] and the ambipolar diffusion theory of SCHOTTKY [2]. The first is usually applied if the mean free path for ion-neutral particle collisions is larger than the capillary radius, the second is used if the pressure is high enough that the ion mean free path is much smaller than the capillary radius. Both theories regard the plasma as quasineutral, i.e. the plasma boundary sheath is not considered. The Tonks-Langmuir theory leads mathematically to a plasma-sheath boundary at a finite radius, where both the plasma density and the potential are finite but their gradients become infinitely large. The ambipolar diffusion theory on the other hand, does not lead to a plasma boundary sheath, the plasma density becomes zero whereas the potential and the ion velocity are infinitely high at a finite radius.

It has been shown by PERsson [3] that the consideration of a (nonlinear) inertia term in the ion momentum balance equation changes the ambipolar diffusion theory in such a way that a plasma sheath boundary exists for which the plasma density and the potential both remain finite. SELF and EwALD [4] pointed out, that by taking into account the inertia term, the 
Schottky theory can also be applied for low pressures and leads to results which are in good agreement with those of the free fall theory. Thus, the Self-Ewald approach represented a transition between the two older theories and provided results also for intermediate pressures. In further publications of FORREST and FRANKLIN [5, 6] a hydrodynamical description of the positive column was used and quasi-neutrality was assumed throughout the work. This resulted in an effective one-fluid model of a gas of particles with mass $m:=m_{i}+m_{e}$, temperature $T:=T_{e}+T_{i}$ and average velocity $v:=v_{i}=v_{e}$. In this model a singularity of the derivatives of the drift velocity and the particle density occurs when the drift velocity reaches the so called Bohm velocity (BoHM [7])

$$
v_{\text {Bohm }}=\sqrt{\frac{k_{B}\left(T_{e}+T_{i}\right)}{m_{e}+m_{i}}} .
$$

The singularity occurs near the wall and signifies the end of the quasineutral region and the beginning of the plasma boundary sheath. In the one-fluid model, the singularity is frequently used as the boundary of the positive column when the width of the sheath is much smaller than the extension of the column itself (Persson [3], Kino, Shaw [8], Forrest, FrankLin [5], VALENTINI [9]).

Although the charge density is much smaller within the column than in the boundary sheath, its correct modelling in the whole plasma is necessary in order to achieve a detailed description of the plasma-sheath boundary and of the sheath itself. Thus, FRIEDMAN and LEVI [10], Ingold [11], Valentini [12,13], and MeTZE, ERnie and Oskam [14] abandoned the assumption of quasi-neutrality and developed a two-fluid model, in which no derivative shows a singularity at the Bohm velocity. However, two new singularities arise at the isothermal ion and electron sonic velocities

$$
v_{i s}=\sqrt{\frac{k_{B} T_{i}}{m_{i}}}, \quad v_{e s}=\sqrt{\frac{k_{B} T_{e}}{m_{e}}} .
$$

While the electrons do not reach the sonic velocity within the plasma, the ion velocity of sound is reached within the positive column. Since the approximate quasi-neutral solution provides continuous curves for density and drift velocity at those points, FRIEDMAN and LEVI [10], INGOLD [11], FrankLIN [15] and VALENTINI [12,13] postulated that the singularity in the two-fluid model must be removable. FRIEDMAN and LEVI [10] therefore assumed that the ratio of ion and electron densities on the axis cannot be chosen arbitrarily but is fixed by an internal condition at the ion sonic point. Their internal condition was $n_{i}=n_{e}$ which, however, leads to physically unreasonable results. INGOLD [11] used $T_{i}=0$ and achieved that the critical point is located at the cylinder axis where it can easily be removed by a Taylor series expansion. The assumption $T_{i}=0$ is not needed in the present treatment.

In all the papers mentioned above the assumption of a neutral gas at rest with constant density was made which is reasonable if the degree of ionization is low. But in the case of a high degree of ionization the ionization processes cause a non-constant neutral gas density

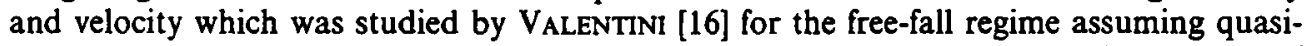
neutrality. We worked with a three-fluid model from which we can infer the radial behavior of the density and the average velocity of neutral atoms and took into account all elastic collisions between the plasma particles and Coulomb collisions between electrons and ions.

The frequently used assumption of a constant ion temperature was dropped in papers of ILIC [17], VALENTINI [18] and WALLSCHLÄGER [19]. While ILIC, however, in his quasi-neutral model did not take into account the heat fluxes, VALENTINI in his two-fluid model put $T_{i}=0$ on the axis. WALLSCHLÄGER in his kinetic treatment considered variable ion temperature but assumed a cold neutral gas $\left(T_{n} \approx 0\right)$. The radial variation of the electron temperature was 
calculated by VALENTINI [13] by means of a two-fluid model including the energy balance of the electrons but assuming constant ion temperature.

In the present paper, in addition to particle densities and average velocities also the radial dependence of temperatures and heat fluxes of electrons, ions and neutrals are studied. Our model assumptions are introduced in section 2.1 . The radial balance equations of particle densities, momentum, energy and the equations for the heat fluxes and Poisson's equation lead to a system of 13 coupled differential equations (section 2.2). The dimensionless form of the equations, which we need for the numerical treatment, is shown in section 2.3. Furthermore we discuss the singularities of the system of equations (section 2.4) and its boundary conditions (section 2.5). The material functions needed for the simulation are presented in section 3. In section 4 we describe the numerical method of solution, a relaxation method for boundary value problems (HENYEY et al. [20], NOBILI, TUROLLA [21]), which can also handle internal conditions at singular critical points. The results obtained for a typical $\mathrm{Ar}^{+}$-laser plasma at electron temperatures between 30,000 and $50,000 \mathrm{~K}$ are discussed in section 5 .

\section{Model Assumptions, Differential Equations and Boundary Conditions}

In this section we will briefly describe the geometry of the discharge, the simplifying assumptions, the hydrodynamic equations and the boundary conditions of such a model system.

\subsection{Model Assumptions}

The geometrical situation is sketched in Fig. 1. The length $l$ of the capillary is of the order of $0.5 \mathrm{~m}$ and the radius $R$ is about $1 \mathrm{~mm}$. A typical value of the gas pressure $p_{n}$ is 1 Torr, the current $I$ in axial direction varies between 5 and $50 \mathrm{~A}$, and the longitudinal electric field strength $E_{z}$ between 5 and $10 \mathrm{Vcm}^{-1}$. The number density of the neutral gas $n_{n}$ covers the range between $3 \cdot 10^{15}$ and $2 \cdot 10^{16} \mathrm{~cm}^{-3}$. The temperature of the neutral gas $T_{n}$ and of the ions $T_{i}$ varies between 500 and $3,000 \mathrm{~K}$ and the temperature of the electrons $T_{e}$ is in the range from 30,000 to $50,000 \mathrm{~K}$.

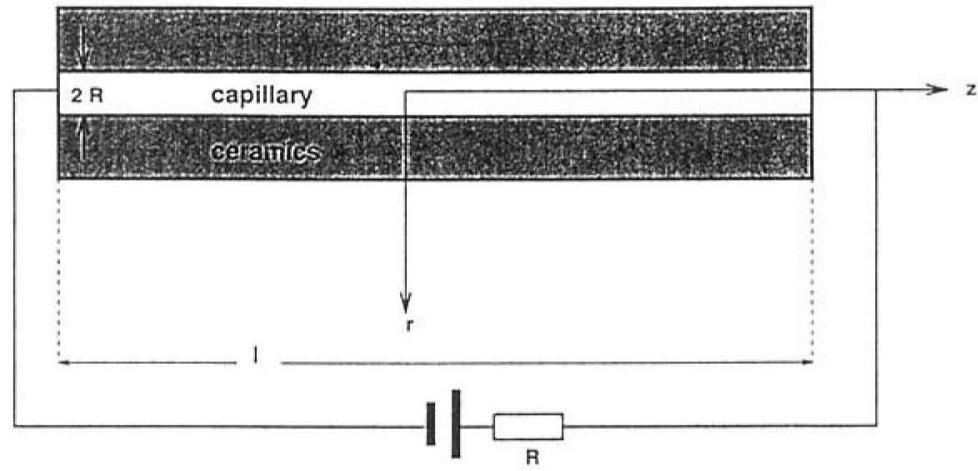

Fig. 1: The geometrical situation of the argon ion laser. The length / of the capillary is of the order of $0.5 \mathrm{~m}$ and the radius $R$ about $1 \mathrm{~mm}$.

Due to the axial symmetry the use of cylindrical coordinates $r, \varphi, z$ is appropriate and none of the quantities depends on $\varphi$. Furthermore, our model is restricted to the region of the positive column which represents the active medium of a laser. In this region with a sufficiently large distance to the anode and cathode, no gradients in axial direction occur and, consequently, all plasma parameters depend only on the radial coordinate $r$. 
The plasma in the column is produced by ionization due to electron collisions. For reasons of simplicity, the plasma is assumed to consist of argon atoms in the ground state, argon ions in the ground state and electrons. Atoms and ions in excited states are disregarded as well as contributions to ionization from excited states which can be estimated to be $\leq 30 \%$ (TIEMANN [22], SCHWAB, KöHLER [23]). Therefore, with regard to ionization the present theory represents a zeroth order approach. The recombination of electrons and ions to neutral atoms is assumed to take place at the wall of the tube. Three-particle recombination in the bulk plasma is neglected.

\subsection{Equations}

The plasma is described by a three-fluid model for the electrons $\left(q_{e}=-e\right)$, the ions $\left(q_{i}=e\right)$ and the neutral atoms. The unknown quantities are the densities $n_{e}, n_{i}, n_{n}$, the mean velocities $v_{e}, v_{i}, v_{n}$, the temperatures $T_{e}, T_{i}, T_{n}$, the heat currents $q_{e}, q_{i}, q_{n}$, and the radial component of the electric field $E_{r}$. The goal of the numerical simulation is to calculate the radial dependence of these quantities, by integrating the hydrodynamic equations together with the Poisson equation. They can be derived from the Boltzmann equation in a standard manner (MITCHNER, KRUGER [24]). For the stationary case these equations are:

The continuity equations:

$$
\begin{aligned}
& \nabla \cdot\left(n_{e} v_{e}\right)=n_{e} v_{i o n}, \\
& \nabla \cdot\left(n_{i} v_{i}\right)=n_{e} v_{i o n}, \\
& \nabla \cdot\left(n_{n} v_{n}\right)=-n_{e} v_{i o n} .
\end{aligned}
$$

The momentum balance equations:

$$
\begin{aligned}
& n_{e} m_{e}\left(v_{e} \cdot \nabla\right) v_{e}+m_{e} n_{e} v_{i o n} v_{e}+\nabla\left(n_{e} k_{B} T_{e}\right)+n_{e} e E= \\
& v_{e n} n_{e} m_{e n}^{*}\left(v_{n}-v_{e}\right)+v_{e i} n_{e} m_{e i}^{*}\left(v_{i}-v_{e}\right), \\
& n_{i} m_{i}\left(v_{i} \cdot \nabla\right) v_{i}+m_{i} n_{e} v_{i o n} v_{i}+\nabla\left(n_{i} k_{B} T_{i}\right)-n_{i} e E= \\
& v_{i n} n_{i} m_{i n}^{*}\left(v_{n}-v_{i}\right)+v_{i e} n_{i} m_{i e}^{*}\left(v_{e}-v_{i}\right), \\
& n_{n} m_{n}\left(v_{n} \cdot \nabla\right) v_{n}-m_{n} n_{e} v_{i o n} v_{n}+\nabla\left(n_{n} k_{B} T_{n}\right)= \\
& +v_{n e} n_{n} m_{n e}^{*}\left(v_{e}-v_{n}\right)+v_{n i} n_{n} m_{n i}^{*}\left(v_{i}-v_{n}\right) .
\end{aligned}
$$

The energy equations:

$$
\begin{aligned}
& \nabla \cdot\left(\left(\frac{5}{2} k_{B} T_{e}+\frac{1}{2} m_{e} v_{e}^{2}\right) n_{e} v_{e}\right)+\nabla \cdot q_{e}+e n_{e} v_{e} \cdot E \\
& =\frac{3}{2} k_{B}\left(T_{n}-T_{e}\right) 2 \frac{m_{e}}{m_{n}} n_{e} v_{e n}+\frac{3}{2} k_{B}\left(T_{i}-T_{e}\right) 2 \frac{m_{e}}{m_{i}} n_{e} v_{e i}-n_{e} \varepsilon_{i o n} v_{i o n}, \\
& \nabla \cdot\left(\left(\frac{5}{2} k_{B} T_{i}+\frac{1}{2} m_{i} v_{i}^{2}\right) n_{i} v_{i}\right)+\nabla \cdot q_{i}-e n_{i} v_{i} \cdot E \\
& =\frac{3}{2} k_{B}\left(T_{n}-T_{i}\right) 2 \frac{m_{i}}{m_{n}} n_{i} v_{i n}+\frac{3}{2} k_{B}\left(T_{e}-T_{i}\right) 2 \frac{m_{e}}{m_{i}} n_{i} v_{i e},
\end{aligned}
$$




$$
\begin{aligned}
& \nabla \cdot\left(\left(\frac{2}{5} k_{B} T_{n}+\frac{1}{2} m_{n} v_{n}^{2}\right) n_{n} v_{n}\right)+\nabla \cdot q_{n} \\
& =\frac{3}{2} k_{B}\left(T_{e}-T_{n}\right) 2 \frac{m_{e}}{m_{n}} n_{n} v_{n e}+\frac{3}{2} k_{B}\left(T_{i}-T_{n}\right) 2 n_{n} v_{n i} .
\end{aligned}
$$

The heat current of the electrons:

$$
q_{e}=-\lambda_{e} \nabla T_{e} .
$$

The heat currents of the ions and atoms:

$$
\begin{aligned}
& q_{i}=-\lambda_{i i} \nabla T_{i}-\lambda_{i n} \nabla T_{n}, \\
& q_{n}=-\lambda_{n i} \nabla T_{i}-\lambda_{n n} \nabla T_{n} .
\end{aligned}
$$

The Poisson equation:

$$
\nabla \cdot E=\frac{e}{\varepsilon_{0}}\left(n_{i}-n_{e}\right) .
$$

Here, $v_{i o n}$ denotes the ionization frequency, $\varepsilon_{i o n}$ the ionization energy of neutral atoms in the ground state, $v_{a b}$ the averaged collision frequency for the momentum transfer between the corresponding constituents, $m_{a b}^{*}=m_{a} m_{b} /\left(m_{a}+m_{b}\right)$ the reduced masses, and $\lambda_{e}, \lambda_{a b}$ the heat conductivity coefficients. The continuity equations have been used to rewrite the inertia terms in the momentum equations and the viscous pressure terms have been neglected.

Instead of the collision frequencies $v_{i o n}, v_{a b}$ rate coefficients $s_{i o n}, s_{e i}, s_{e n}, s_{i n}$ are introduced by the following relations:

$$
\begin{aligned}
& v_{i o n}=n_{n} s_{i o n}, \\
& v_{e i}=\frac{n_{i}}{n_{e}} v_{i e}=n_{i} s_{e i}, \\
& v_{e n}=\frac{n_{n}}{n_{e}} v_{n e}=n_{n} s_{e n}, \\
& v_{i n}=\frac{n_{n}}{n_{i}} v_{n i}=n_{n} s_{i n} .
\end{aligned}
$$

Furthermore, by using Ohm's law, the Joule heating terms in the energy equations can be written as:

$$
\begin{aligned}
-e n_{e} v_{e} \cdot E & =-e n_{e} v_{e} E_{r}+\sigma_{e} E_{z}^{2}, \\
e n_{i} v_{i} \cdot E & =e n_{i} v_{i} E_{r}+\sigma_{i} E_{z}^{2} .
\end{aligned}
$$

The velocities have been decomposed into the radial components $v_{e}, v_{i}$ and the longitudinal components, which are expressed by means of the electrical conductivities $\sigma_{e}, \sigma_{i}$. Due to the approximate spatial homogeneity in axial direction, only the radial components of the vectorial equations have to be taken into account.

\subsection{Dimensionless Equations}

Because of $\mathrm{m}_{n} \approx m_{i}$ and $m_{e} \ll m_{n}$, the notation $M:=m_{n} \approx m_{i}, m_{e}+M \approx M$ will be used.

For the numerical treatment it is appropriate to introduce dimensionless quantities. The 
radial coordinate will be scaled with the radius of the capillary tube:

$$
r=R x \text {. }
$$

The derivative with respect to the dimensionless coordinate $x$ is denoted by a prime. Furthermore we introduce the mass ratio

$$
\mu=m_{e} / M,
$$

the dimensionless densities $N_{a}\left(n_{a}\right.$ in units of the electron density on the axis, $\left.n_{e 0}\right)$

$$
n_{a}=n_{e 0} N_{a},
$$

the dimensionless velocities $V_{a}$

$$
v_{a}=\sqrt{\frac{\varepsilon_{\text {ion }}}{M}} V_{a},
$$

the dimensionless temperatures $\tau_{a}$

$$
k_{B} T_{a}=\varepsilon_{i o n} \tau_{a},
$$

the dimensionless heat currents $Q_{a}$

$$
q_{a}=\varepsilon_{i o n} n_{e 0} \sqrt{\frac{\varepsilon_{i o n}}{M}} Q_{a}
$$

and the dimensionless electric field components $\eta_{r}, \eta_{z}$

$$
E_{r}=\frac{\varepsilon_{\text {ion }}}{e R} \eta_{r}, \quad E_{z}=\frac{\varepsilon_{\text {ion }}}{e R} \eta_{z} .
$$

Using eqs. (20-28), we end up with the following set of 13 coupled first order differential equations:

$$
\begin{aligned}
& V_{e} N_{e}^{\prime}+N_{e} V_{e}^{\prime}=-\frac{1}{x} N_{e} V_{e}+N_{e} N_{n} S_{i o n}, \\
& V_{i} N_{i}^{\prime}+N_{i} V_{i}^{\prime}=-\frac{1}{x} N_{i} V_{i}+N_{e} N_{n} S_{i o n}, \\
& V_{n} N_{n}^{\prime}+N_{n} V_{n}^{\prime}=-\frac{1}{x} N_{n} V_{n}-N_{e} N_{n} S_{i o n}, \\
& \tau_{e} N_{e}^{\prime}+\mu N_{e} V_{e} V_{e}^{\prime}+N_{e} \tau_{e}^{\prime}=-N_{e} \eta_{r}-\mu V_{e} N_{e} N_{n} S_{i o n} \\
& \quad-\mu N_{e} N_{n}\left(V_{e}-V_{n}\right) S_{e n}-\mu N_{e} N_{i}\left(V_{e}-V_{i}\right) S_{e i}, \\
& \tau_{i} N_{i}^{\prime}+N_{i} V_{i} V_{i}^{\prime}+N_{i} \tau_{n}^{\prime}=+N_{i} \eta_{r}-V_{i} N_{e} N_{n} S_{i o n} \\
& \quad-\frac{1}{2} N_{i} N_{n}\left(V_{i}-V_{n}\right) S_{i n}+\mu N_{e} N_{i}\left(V_{e}-V_{i}\right) S_{e i}, \\
& \tau_{n} N_{n}^{\prime}+N_{n} V_{n} V_{n}^{\prime}+N_{n} \tau_{n}^{\prime}=V_{n} N_{e} N_{n} S_{i o n} \\
& \quad+\mu N_{e} N_{n}\left(V_{e}-V_{n}\right) S_{e n}+\frac{1}{2} N_{i} N_{n}\left(V_{i}-V_{n}\right) S_{i n},
\end{aligned}
$$




$$
\begin{aligned}
& \mu N_{e} V_{e}^{2} V_{e}^{\prime}+\frac{5}{2} N_{e} V_{e} \tau_{e}^{\prime}+Q_{e}^{\prime}=-\frac{5}{2} \tau_{e} N_{e} N_{n} S_{i o n}-N_{e} N_{n} S_{i o n} \\
&-\frac{1}{2} \mu V_{e}^{2} N_{e} N_{n} S_{i o n}-\frac{1}{x} Q_{e}-N_{e} V_{e} \eta_{r}+\mu_{e} \eta_{z}^{2} \\
&-3 \mu N_{e} N_{n}\left(\tau_{e}-\tau_{n}\right) S_{e n}-3 \mu N_{e} N_{i}\left(\tau_{e}-\tau_{i}\right) S_{e i} \\
& N_{i} V_{i}^{2} V_{i}^{\prime}+\frac{5}{2} N_{i} V_{i} \tau_{i}^{\prime}+Q_{i}^{\prime}=-\frac{5}{2} \tau_{i} N_{e} N_{n} S_{i o n} \\
&-\frac{1}{2} V_{i}^{2} N_{e} N_{n} S_{i o n}-\frac{1}{x} Q_{i}+N_{i} V_{i} \eta_{r}+\mu_{i} \eta_{z}^{2} \\
&-3 N_{i} N_{n}\left(\tau_{i}-\tau_{n}\right) S_{i n}+3 \mu N_{e} N_{i}\left(\tau_{e}-\tau_{i}\right) S_{e i}, \\
& N_{n} V_{n}^{2} V_{n}^{\prime}+ \frac{5}{2} N_{n} V_{n} \tau_{n}^{\prime}+Q_{n}^{\prime}=\frac{5}{2} \tau_{n} N_{e} N_{n} S_{i o n} \\
&+\frac{1}{2} V_{n}^{2} N_{e} N_{n} S_{i o n}-\frac{1}{x} Q_{n}+3 \mu N_{e} N_{n}\left(\tau_{e}-\tau_{n}\right) S_{e n} \\
&+3 N_{i} N_{n}\left(\tau_{i}-\tau_{n}\right) S_{i n}, \\
& \eta_{r}^{\prime}=-\frac{1}{x} \eta_{r}+C\left(N_{i}-N_{e}\right) \\
& l_{e} \tau_{e}^{\prime}=- Q_{e}, \\
& l_{i i} \tau_{i}^{\prime}+l_{i n}^{\prime} \tau_{n}^{\prime}=-Q_{n n} \tau_{n}^{\prime}=-Q_{n}, \\
& 1
\end{aligned}
$$

where additionally the following dimensionless constants and material functions have been introduced:

$$
\begin{aligned}
& C=\frac{n_{e 0} R^{2} e^{2}}{\varepsilon_{0} \varepsilon_{i o n}} \\
& S_{\alpha}=n_{e 0} R \sqrt{\frac{M}{\varepsilon_{i o n}}} s_{\alpha} \text { with } \alpha=\text { ion, en, in, ei, } \\
& \mu_{\alpha}=\frac{\varepsilon_{i o n}}{n_{e 0} R e^{2}} \sqrt{\frac{M}{\varepsilon_{i o n}}} \sigma_{\alpha} \text { with } \alpha=e, i, \\
& l_{\alpha}=\frac{1}{n_{e 0} R k_{B}} \sqrt{\frac{M}{\varepsilon_{i o n}}} \lambda_{\alpha} \text { with } \alpha=e, i i, i n, n n .
\end{aligned}
$$

\subsection{Singularities}

The 13 first order ordinary differential equations we have to solve represent a quasilinearimplicit system, which can be written in the following matrix form (STOER, BULIRSCH [25]):

$$
U(y) y^{\prime}(x)=t(x, y(x))
$$


with

$$
y^{T}=\left(Q_{e}, Q_{i}, Q_{n}, V_{e}, V_{i}, V_{n}, \eta_{r}, N_{e}, N_{i}, N_{n}, \tau_{e}, \tau_{i}, \tau_{n}\right),
$$

where the superscript $T$ denotes the transposed vector. The components of $y^{\prime}$ can become infinite at those points, where the matrix $U$ is singular, i.e. where

$$
\operatorname{det} U=l_{e} N_{e} N_{i} N_{n}\left(\frac{m}{M} V_{e}^{2}-\tau_{e}\right)\left(V_{i}^{2}-\tau_{i}\right)\left(V_{n}^{2}-\tau_{n}\right)\left(l_{i i} l_{m n}-l_{i n}^{2}\right)=0 .
$$

A singularity is encountered when the drift velocity of the electrons reaches the sonic velocity of the electrons

$$
\left|V_{e s}\right|=\sqrt{\frac{M}{m} \tau_{e}} \text { i.e. }\left|v_{e s}\right|=\sqrt{\frac{k_{B} T_{e}}{m}},
$$

but the electrons do not reach this velocity within the positive column. Another singular point occurs for

$$
\left|V_{n s}\right|=\sqrt{\tau_{n}} \quad \text { i.e. }\left|v_{n s}\right|=\sqrt{\frac{k_{B} T_{n}}{M}} .
$$

However, since we did not exceed $T_{n}=3,000 \mathrm{~K}$ in our calculations, the velocity of the argon atoms does not reach the velocity of sound within the domain of integration.

Since according to the second law of thermodynamics the term $l_{i i} l_{n n}-l_{i n}^{2}$ is always positive, the only critical point of interest occurs if

$$
\left|V_{i s}\right|=\sqrt{\tau_{i}} \text { i.e. }\left|v_{i s}\right|=\sqrt{\frac{k_{B} T_{i}}{M}},
$$

that is at the point where the drift velocity of the ions reaches the ion sonic velocity. This point lies inside our integration domain. But this singularity must be a removable one as has been explained by several authors before (FRANKLIN [15], VALENTINI [12]).

Another virtual singular point lies at the symmetry axis where the right hand side of (46) is undefined, caused by the term $1 / x$. Therefore, we start from the axis with a Taylor series expansion, thus getting rid of any singular behaviour for $x=0$.

\subsection{Boundary Conditions}

Seven of the 13 boundary conditions, which are necessary for the 13 equations cannot be choosen freely but are determined by symmetry reasons. The drift velocities, the heat currents and the radial component of the electrical field must vanish on the axis:

$$
\begin{aligned}
& V_{e}(x=0)=0, \\
& V_{i}(x=0)=0, \\
& V_{n}(x=0)=0, \\
& Q_{e}(x=0)=0, \\
& Q_{i}(x=0)=0, \\
& Q_{n}(x=0)=0, \\
& \eta_{r}(x=0)=0 .
\end{aligned}
$$


Furthermore, our choice of the dimensionless variables leads to

$$
N_{e}(x=0)=n_{e 0} / n_{e 0}=1 \text {, }
$$

introducing $n_{e 0}$ as a parameter of the system. For a given parameter $n_{e 0}$, however, the ion density on the axis can no longer be chosen freely, but is fixed by the postulation of a removable singularity at the ion sonic velocity (FRIEDMAN, LEVI [10]).

If we solve the system (46) for $s^{\prime}$ we obtain for the derivative of $V_{i}$ :

$$
V_{i}^{\prime}=\frac{D(y, x)}{V_{l}^{2}-\tau_{i}}
$$

with

$$
\begin{aligned}
D(y, x)= & V_{i}\left(-\frac{1}{x} N_{i} V_{i}+N_{e} N_{n} S_{i o n}\right. \\
& -\left(N_{i} \eta_{r}-V_{i} N_{e} N_{n} S_{i o n}-\frac{1}{2} N_{i} N_{n}\left(V_{i}-V_{n}\right) S_{i n}\right. \\
& \left.+\mu N_{e} N_{i}\left(V_{e}-V_{i} S_{e i}\right)\right)+N_{i} \tau_{i}^{\prime}
\end{aligned}
$$

The quasi-neutral approximation does not show a singularity at the ion sonic velocity and only a smooth solution does make sense physically. Therefore the singularity must be removable by the l'Hôspital rule which demands that at the critical point where the denominator is zero, also the numerator has to vanish. This postulate leads to an internal boundary condition or regularity condition at the critical point $x_{i s}$ :

$$
D\left(y, x=x_{i s}\right)=0 .
$$

Near the radius of the capillary the electron velocity reaches the thermal value $\left(\frac{k_{B} T_{e}}{2 \pi m}\right)^{1 / 2}$ (INGOLD [11], FRANKLIN [15], Metze et al. [14]). We use the boundary condition suggested by VALENTINI [16]

$$
V_{e}(x=1)=\sqrt{\tau_{e}}, \quad v_{e}\left(r=R_{\mathrm{Bohm}}\right)=\sqrt{\frac{k_{B} T_{e}}{M}},
$$

which overcomes the numerical difficulties introduced by the steep gradients of the electron velocity and the electric field by neglecting the extent of the plasma sheath compared to the capillary radius.

Besides the parameter $n_{e 0}$, only the temperatures $\tau_{e}, \tau_{i}$ and $\tau_{n}$ can be freely chosen at the boundary. We fixed the temperatures at $x=R$ :

$$
\begin{aligned}
& \tau_{e}(x=R)=\tau_{e R}, \\
& \tau_{i}(x=R)=\tau_{i R}, \\
& \tau_{n}(x=R)=\tau_{n R} .
\end{aligned}
$$

\section{Material Characteristics}

For a realistic modelling the knowledge of the correct material properties is essential. Therefore, in this section all material functions as derived from kinetic theory are discussed. 


\subsection{Ionization Rate}

The rate coefficient for the ionization of argon $s_{i o n}$ is obtained from the ionization cross section $\sigma_{i o n}$ by averaging

$$
s_{\text {ion }}=\left\langle\sigma_{\text {ion }} v\right\rangle
$$

with the normalized electron energy distribution function $f_{0}(\varepsilon)$ :

$$
\left\langle\sigma_{i o n} v\right\rangle=\int_{0}^{\infty} \sqrt{\frac{2 \varepsilon}{m_{e}}} \sigma_{i o n} \sqrt{\varepsilon} f_{0}(\varepsilon) \mathrm{d} \varepsilon .
$$

In Fig. 2, the experimentally determined ionization cross section $\sigma_{\text {ion }}$ (STEPHAN, HELM, MÄRK [26]) is shown in its dependence on the electron energy $\varepsilon$. Using a Maxwell distribution

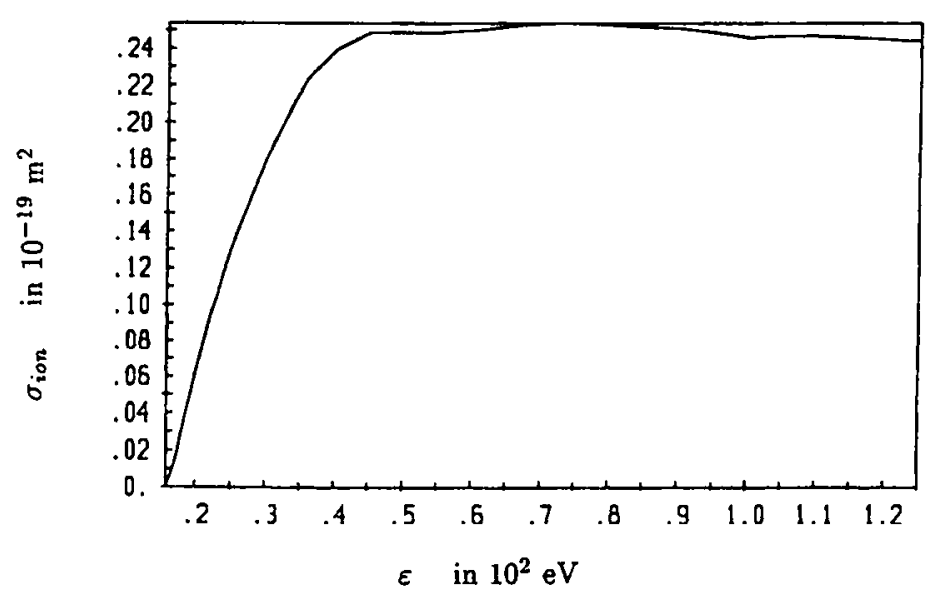

Fig. 2: The ionization cross section $\sigma_{i o n}$ of argon from the ground state as a function of the electron energy $\varepsilon$ (STEPHAN et al., 1980).

$$
f_{0}(\varepsilon)=\frac{2}{\sqrt{\pi}}\left(k_{B} T_{e}\right)^{-3 / 2} \exp \left(-\frac{\varepsilon}{k_{B} T_{e}}\right) \text { with } \int_{0}^{\infty} f_{0}(\varepsilon) \varepsilon^{1 / 2} \mathrm{~d} \varepsilon=1,
$$

which is a good approximation because of the high degree of ionization $(\alpha \lesssim 0.01)$, the ionization rate coefficient $s_{i o n}$ can be calculated as a function of the electron temperature $T_{e}$. The result is shown in Fig. 3.

\subsection{Collision Rates}

In Fig. 4, the experimentally determined momentum transfer cross section $\sigma_{m o m}^{e n}$ for collisions between electrons and neutral argon atoms (KRENZ [27]) is shown in its dependence on the electron energy $\varepsilon$. Using again a Maxwell distribution the rate coefficient

$$
s_{e n}=\left\langle\sigma_{\text {mom }}^{e n} v\right\rangle
$$

can be calculated as a function of the electron temperature $T_{e}$ and is shown in Fig. 5 .

The rate for collisions between ions and atoms is defined as

$$
s_{i n}=\left\langle\sigma_{i n}^{\text {int }} v_{i n}^{\text {rel }}\right\rangle \text {, }
$$




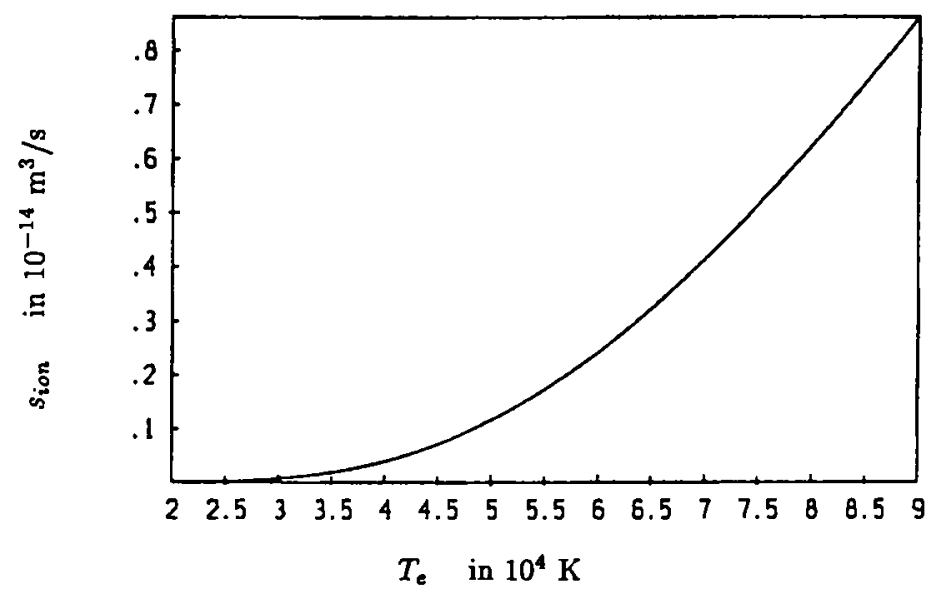

Fig. 3: The rate coefficient $s_{i o n}$ for the ionization of argon from the ground state as a function of the electron temperature $T_{e}$.

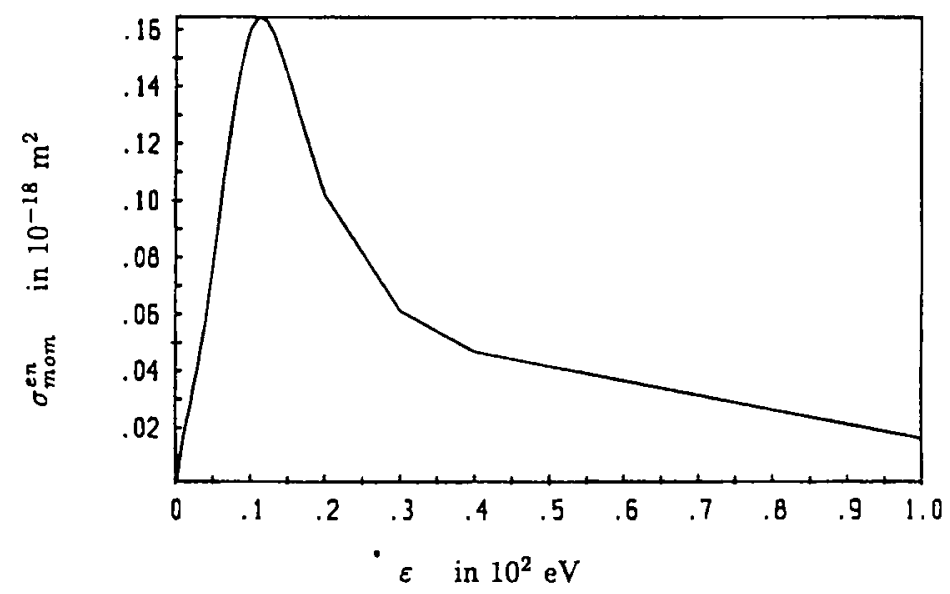

Fig. 4: The momentum transfer cross section $\sigma_{\text {mom }}^{\text {en }}$ for collisions between electrons and neutral argon atoms (KRENZ, 1987) as a function of the electron energy $\varepsilon$.

with

$$
\begin{aligned}
& \left\langle\sigma_{i n}^{\text {tot }} v_{i n}^{\text {rel }}\right\rangle=4 \pi\left(\frac{M}{2 \pi k_{B}\left(T_{i}+T_{n}\right.}\right)^{3 / 2} \\
& \int_{0}^{\infty} \mathrm{d} g g^{3} \mathrm{e}^{-M g^{2} / 2 k\left(T_{i}+T_{n}\right)}\left(2 \pi \int_{0}^{\pi}(1-\cos \vartheta) \sigma_{i n}^{\text {el }}(\vartheta, g) \sin \vartheta d \vartheta\right)
\end{aligned}
$$

and

$$
g=v_{i n}^{\mathrm{rel}} .
$$

Based on the rigid-sphere approximation, where the cross section for elastic collisions is isotropic $\left(\sigma_{i n}^{e l}=d_{i n}^{2} / 4\right)$, one obtains

25 Contrib. Plasma Phys. 32 (1992) 5 


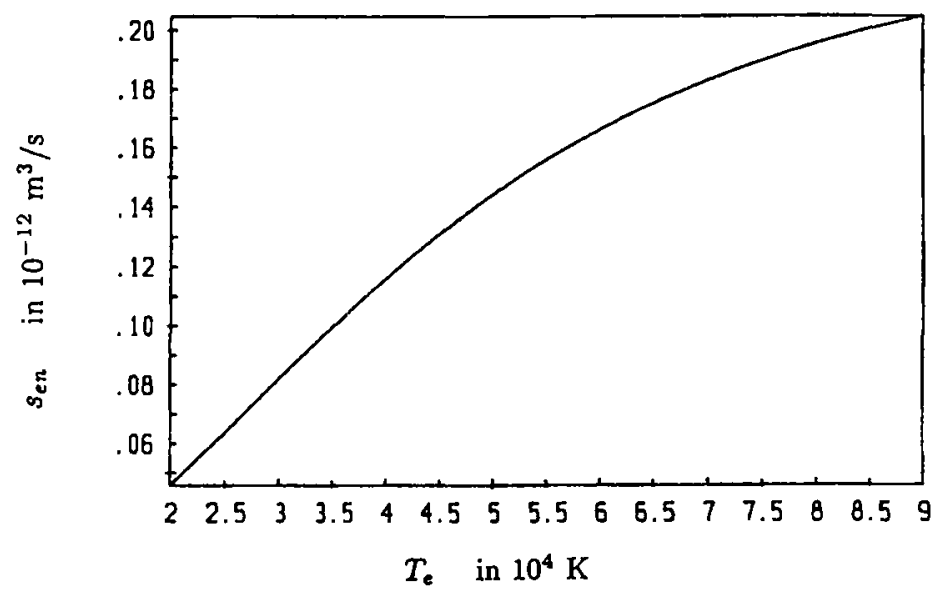

Fig. 5: The rate coefficient $s_{e n}$ for collisions between electrons and neutral argon atoms as a function of the electron temperature $T_{e}$.

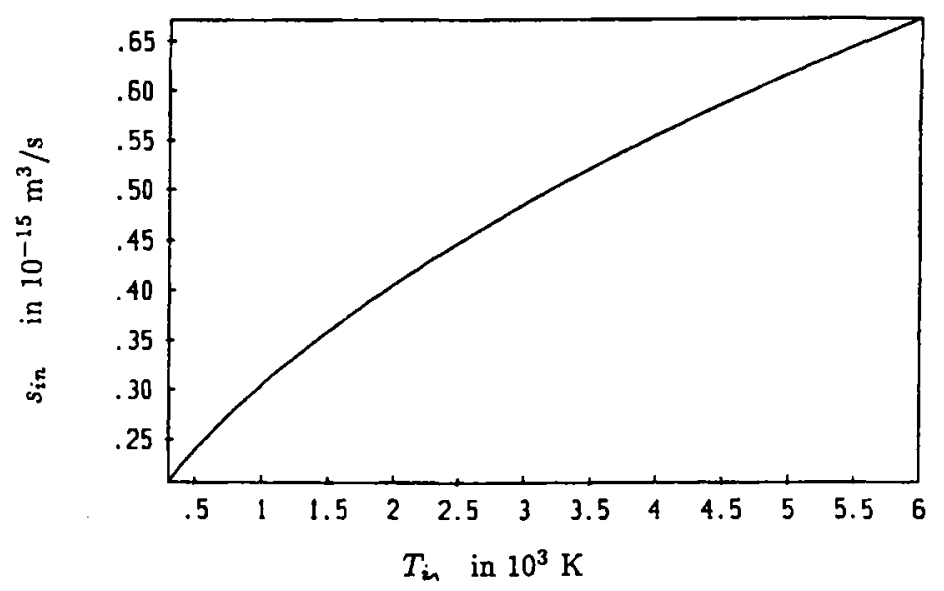

Fig. 6: The rate coefficient $s_{\text {in }}$ for collisions between ions and neutral argon atoms as a function of the gas temperature $T_{n} \approx T_{i}$.

$$
s_{i n}=\left\langle\sigma_{i n}^{\text {tot }} v_{i n}^{\text {rel }}\right\rangle=\sqrt{\frac{8 k_{B}\left(T_{i}+T_{n}\right)}{\pi m_{n}}} \pi d_{i n}^{2}
$$

with $d_{i n}=1 / 2\left(d_{i}+d_{n}\right)$ being the average diameter of the collision partners. The variation of $s_{i n}$ with respect to the gas temperature $T_{n}$ is plotted in Fig. 6 .

The rate coefficient $s_{e i}$ for Coulomb collisions between electrons and ions is [24]

$$
s_{e i}=\frac{4}{3} \sqrt{2 \pi}\left(\frac{m_{e}}{k_{B} T_{e}}\right)^{3 / 2}\left(\frac{e^{2}}{4 \pi \varepsilon_{0} m_{e}}\right)^{2} \ln \Lambda,
$$

where $\ln A$ is the Coulomb logarithm

$$
\ln A=\ln \left(1.239 \cdot 10^{7} T_{e}^{3 / 2} n_{e}^{-1 / 2}\right), \quad T_{e} \text { in } \mathrm{K}, n_{e} \text { in } \mathrm{m}^{-3} .
$$

The dependence of $s_{e i}$ on the electron temperature $T_{e}$ is shown in Fig. 7. 


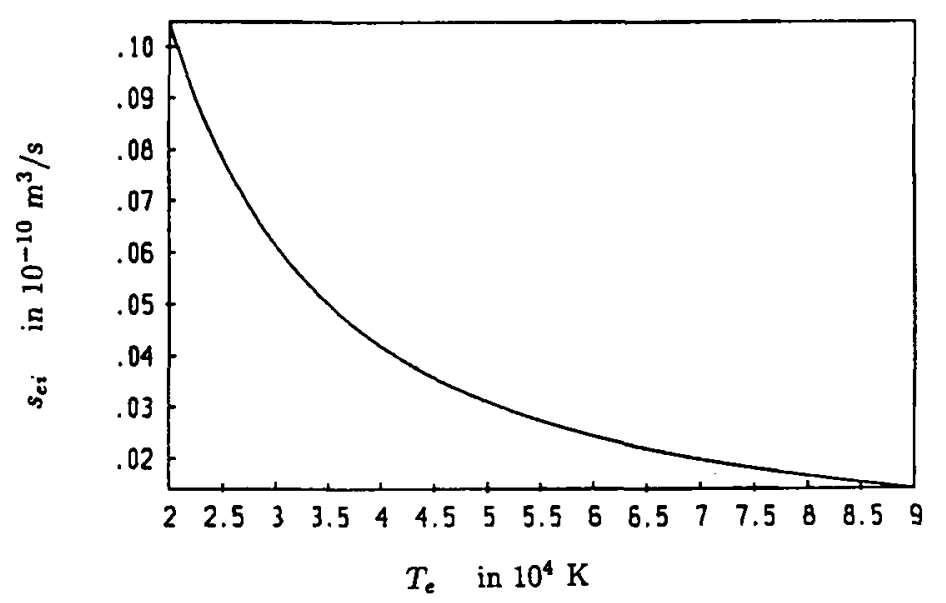

Fig. 7: The rate coefficient $s_{e i}$ for collisions between electrons and ions as a function of the electron temperature $T_{e}$.

\subsection{Electrical Conductivities}

The electrical conductivity of the electrons $\sigma_{e}$ can be calculated from [24]

$$
\sigma_{e}=\frac{2 n_{e} e^{2}}{3 k_{B} T_{e} m_{e}} \int_{0}^{\infty} \frac{\varepsilon^{3 / 2}}{v_{\text {eff }}^{\sigma}(\varepsilon)} f_{0}(\varepsilon) \mathrm{d} \varepsilon
$$

with the effective rate coefficient

$$
\begin{aligned}
v_{\mathrm{efr}}^{o}(\varepsilon)= & n_{n} \sqrt{\frac{2 \varepsilon}{m_{e}}} \sigma_{m o m}^{e n}(\varepsilon)+6.578 \cdot 10^{-29} \varepsilon^{-1} T_{e}^{-1 / 2} n_{i} \ln A \\
& \left(T_{e} \text { in } \mathrm{K}, n_{i} \text { in } \mathrm{m}^{-3} \text { und } \varepsilon \text { in } \mathrm{J}\right) .
\end{aligned}
$$

Using again a Maxwell distribution for $f_{0}(\varepsilon), \sigma_{e}$ is shown in Fig. 8 for electron temperatures between 20,000 und $90,000 \mathrm{~K}$ and $n_{e} / n_{n}=0.01$.

The electrical conductivity for ions is derived from

$$
\sigma_{i}=\frac{3 n_{i} e^{2}}{8 M \Omega_{i n}^{(1,1)} n_{n}},
$$

where $\Omega_{i n}^{(1,1)}$ is a Chapman-Cowling integral [28]. The rigid-sphere approximation results in

$$
\sigma_{i}=n_{i} e^{2}\left(\left(n_{i}+n_{n}\right) M \frac{4}{3} \sqrt{\frac{2 k_{B}\left(T_{i}+T_{n}\right)}{\pi M}} \pi d_{i n}^{2}\right)^{-1},
$$

which is shown in Fig. 9 as a function of the ion temperature $T_{i}\left(=T_{n}\right)$ and for a degree of ionization of $n_{i} / n_{n}=0.01$. 


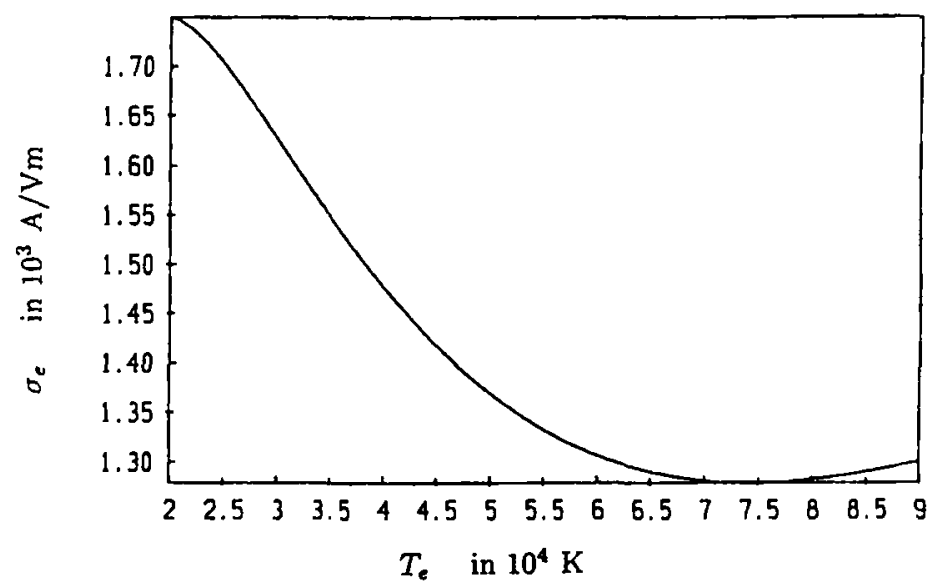

Fig. 8: The electrical conductivity of the electrons $\sigma_{e}$ as a function of the electron temperature $T_{e}$ for a degree of ionization of 0.01 .

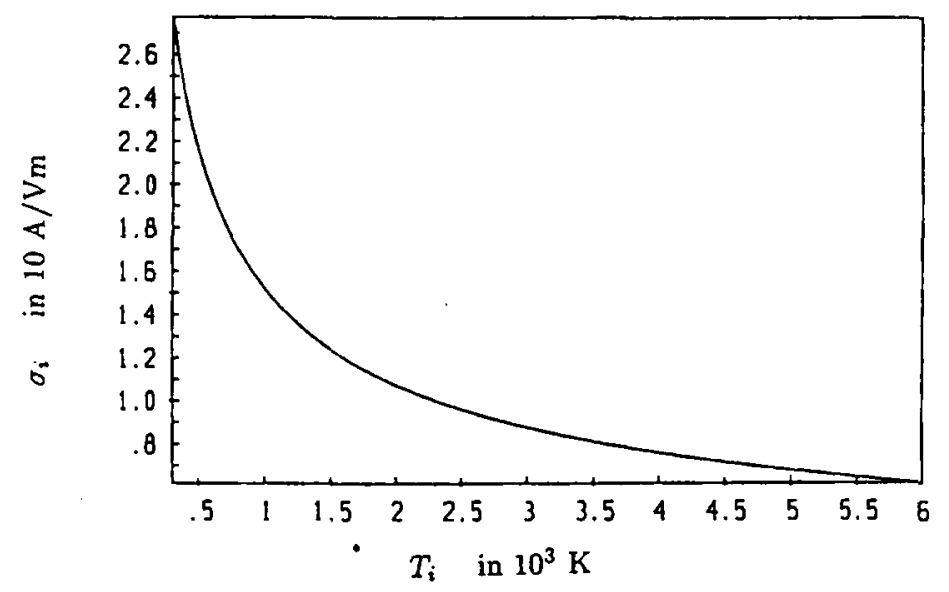

Fig. 9: The electrical conductivity of the ions $\sigma_{i}$ as a function of the ion temperature $T_{i}$ for a degree of ionization of 0.01 and gas temperature $T_{n}=T_{i}$.

\subsection{Thermal Conductivities}

The thermal conductivity of the electrons $\lambda_{e}$ can be calculated from [24]

$$
\lambda_{e}=\frac{2}{3} \frac{n_{e} k_{B}}{m_{e}} \int_{0}^{\infty}\left(\frac{\varepsilon}{k_{B} T_{e}}-\frac{5}{2}\right)^{2} \frac{\varepsilon^{3 / 2}}{\nu_{e f r}^{\lambda}(\varepsilon)} f_{0}(\varepsilon) \mathrm{d} \varepsilon
$$

with the effective collision rate

$$
\begin{aligned}
v_{\text {eff }}^{\lambda}(\varepsilon)= & n_{n} \sqrt{\frac{2 \varepsilon}{m_{e}}} \sigma_{m o m}^{e n}(\varepsilon)+1.349 \cdot 10^{-28} \varepsilon^{-1} T_{e}^{-1 / 2} n_{i} \ln A \\
& \left(T_{e} \text { in } \mathrm{K}, n_{i} \text { in } \mathrm{m}^{-3} \text { und } \varepsilon \text { in } \mathrm{J}\right)
\end{aligned}
$$




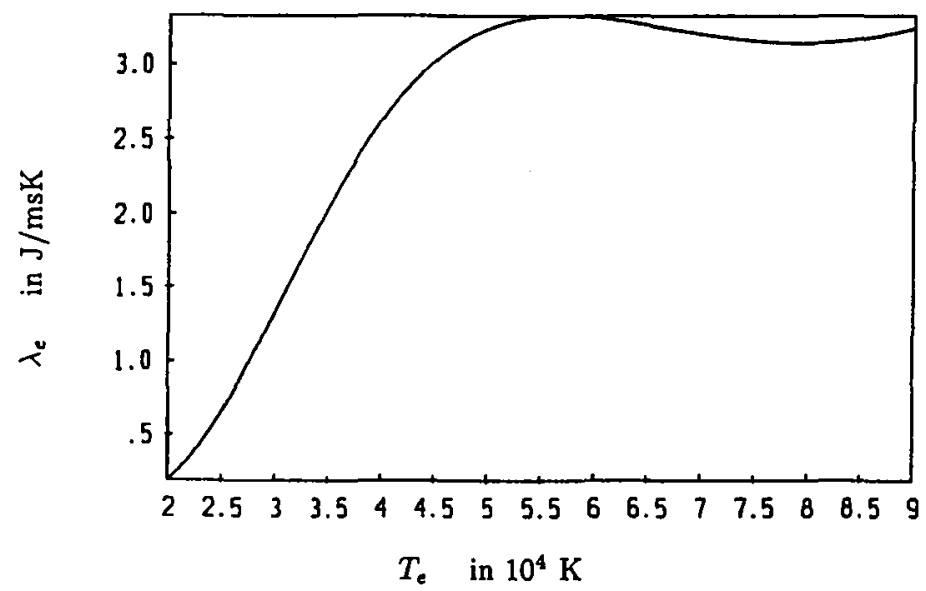

Fig. 10: The thermal conductivity of the electrons $\lambda_{e}$ as a function of the electron temperature $T_{e}$ for $n_{e}=10^{20} \mathrm{~m}^{-3}$.

and is shown in Fig. 10 as a function of the electron temperature $T_{e}$ and for $n_{e}=10^{20} \mathrm{~m}^{-3}$.

The ionic heat current $q_{i}$ is not only determined by $\nabla T_{i}$, but also as a result of the influence of the ion-neutral collisions by $\nabla T_{n}$ and vice versa for the neutral heat current $q_{n}$ (eqs. (13-14)). This coupling is expressed by means of the coefficients $\lambda_{i i}, \lambda_{i n}=\lambda_{n i}$ and $\lambda_{n n}$, which can be derived from the Boltzmann equation by appropriate integration. Based on the approximation $n_{i} \ll n_{n}$ (degree of ionization $\approx 1 \%$ ) they take the form:

$$
\begin{aligned}
& \lambda_{i i}=\frac{5 k_{B}{ }^{2} T_{i}}{2 M} \frac{n_{i}}{n_{n}} \frac{1}{v_{i n} \sigma_{i n}^{(1)}}, \\
& \lambda_{i n}=\lambda_{n i}=-\frac{5 k_{B}{ }^{2}\left(T_{i} T_{n}\right)^{1 / 2}}{2 M} \frac{n_{i}}{n_{n}} \frac{v_{i n} \sigma_{i n}^{(2)}}{v_{i n} \sigma_{i n}^{(1)} v_{n n} \sigma_{n n}}, \\
& \lambda_{n n}=\frac{5 k_{B}{ }^{2} T_{n}}{2 M} \frac{1}{v_{n n} \sigma_{n n}},
\end{aligned}
$$

with

$$
v_{i i}=\sqrt{\frac{16 k_{B} T_{i}}{\pi M}}, \quad v_{n n}=\sqrt{\frac{16 k_{B} T_{n}}{\pi M}}, \quad v_{i n}=\sqrt{\frac{8 k_{B}\left(T_{i}+T_{n}\right)}{\pi M}},
$$

and the effective collision cross sections $\sigma_{i i}, \sigma_{i n}^{(1)}, \sigma_{i n}^{(2)}=\sigma_{i n}^{(2)}, \sigma_{n n}$. The ion-ion collisions are characterized by $\sigma_{i i}$, the ion-neutral collisions by $\sigma_{i n}^{(1)}, \sigma_{i n}^{(2)}$ and the neutral-neutral collisions by $\sigma_{n n}$. These effective collision cross sections can again be obtained from the Chapman-Cowling integrals. Assuming $m_{n}=m_{i}=M$, one finds (MCCOURT, BEENNAKKER, KöHLER and KuSCER [29]):

$$
\begin{aligned}
& v_{n n} \sigma_{n n}=\frac{16}{15} \Omega_{n n}^{(2,2)}, \\
& v_{i n} \sigma_{i n}^{(1)}=\frac{1}{8}\left(\frac{88}{3} \Omega_{i n}^{(1,1)}-\frac{32}{3} \Omega_{i n}^{(1,2)}+\frac{32}{15} \Omega_{i n}^{(1,3)}+\frac{64}{15} \Omega_{i n}^{(2,2)}\right), \\
& v_{i n} \sigma_{i n}^{(2)}=\frac{1}{8}\left(-\frac{88}{3} \Omega_{i n}^{(1,1)}+\frac{32}{3} \Omega_{i n}^{(1,2)}-\frac{32}{15} \Omega_{i n}^{(1,3)}+\frac{64}{15} \Omega_{i n}^{(2,2)}\right) .
\end{aligned}
$$


In rigid-sphere approximation, which is appropriate for high temperatures, the $\Omega$-integrals can be evaluated to give

$$
\begin{aligned}
& \Omega_{i n}^{(1,1)}=\bar{v}_{i n} d_{i n}^{2}, \\
& \Omega_{i n}^{(1,2)}=3 \bar{v}_{i n} d_{i n}^{2}, \\
& \Omega_{i n}^{(1,3)}=12 \bar{v}_{i n} d_{i n}^{2}, \\
& \Omega_{i n}^{(2,2)}=2 \bar{v}_{i n} d_{i n}^{2},
\end{aligned}
$$

with the abbreviation

$$
\bar{v}_{i n}=\sqrt{\frac{\pi k_{B}\left(T_{i}+T_{n}\right)}{2 M}} .
$$

From these equations (82)-(93) the thermal conductivity coefficients for ions and neutral atoms can be written as

$$
\begin{aligned}
& \lambda_{i i}=\frac{5 k_{B}^{2} T_{i}}{2 M}\left(\frac{n_{i}}{n_{n}}\right) \frac{15}{59 d_{i n}^{2}}\left(\frac{\pi k_{B} T_{i n}}{M}\right)^{-1 / 2}, \\
& \lambda_{i n}=\frac{5 k_{B}^{2}\left(T_{i} T_{n}\right)^{1 / 2}}{2 M}\left(\frac{n_{i}}{n_{n}}\right) \frac{405}{1888 d_{n n}^{2}}\left(\frac{\pi k_{B} T_{n}}{M}\right)^{-1 / 2}, \\
& \lambda_{n n}=\frac{5 k_{B}^{2} T_{n}}{2 M} \frac{15}{32 d_{n n}^{2}}\left(\frac{\pi k_{B} T_{n}}{M}\right)^{-1 / 2},
\end{aligned}
$$

with the average temperature $T_{i n}=\left(T_{i}+T_{n}\right) / 2$. They are shown in Fig. 11 to Fig. 13 as functions of the temperature $T_{i}=T_{n}$ and for a degree of ionization $n_{i} / n_{n}=0.01$.

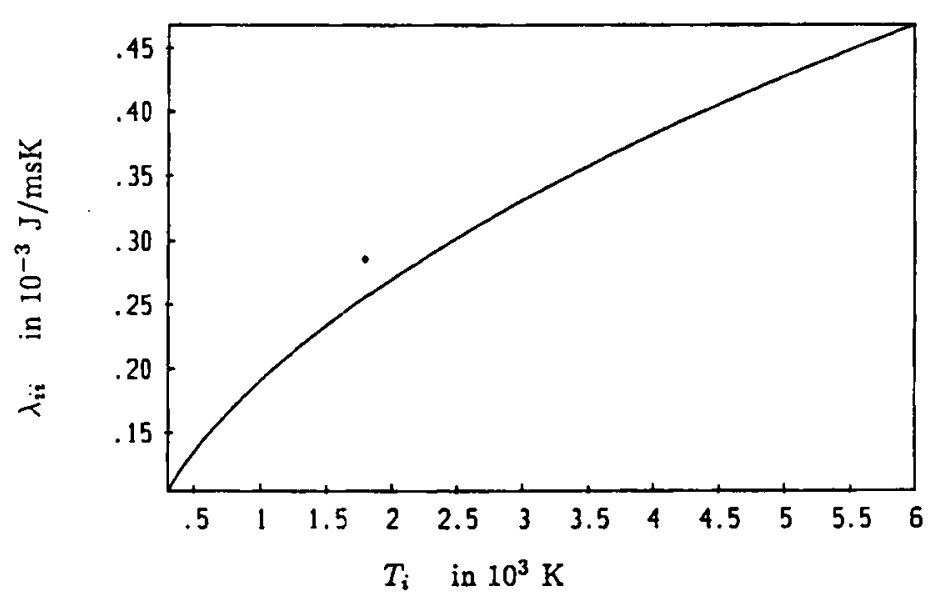

Fig. 11: The thermal conductivity coefficient $\lambda_{i i}$ as a function of the ion temperature $T_{i}=T_{n}$. 


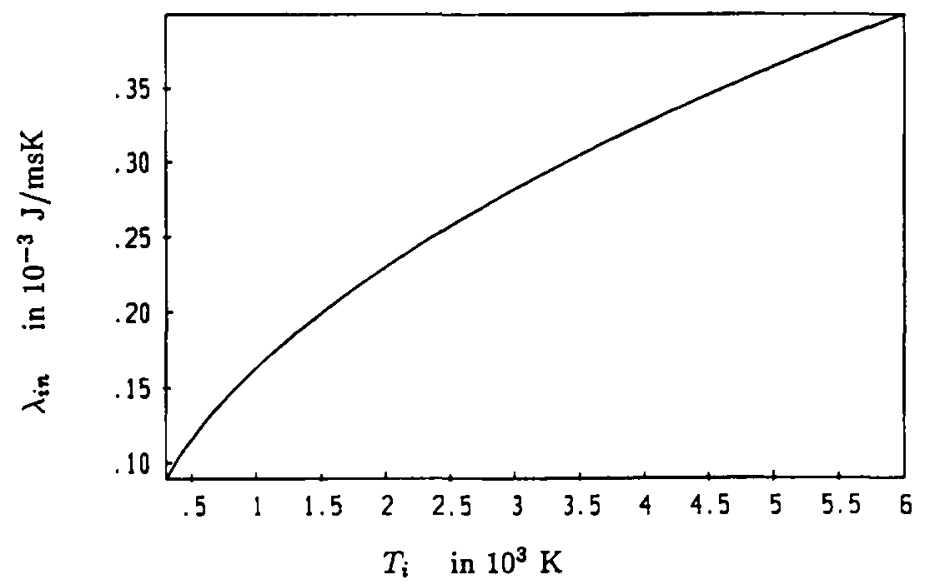

Fig. 12: The thermal conductivity coefficient $\lambda_{\text {in }}$ as a function of the ion temperature $T_{i}=T_{n}$.

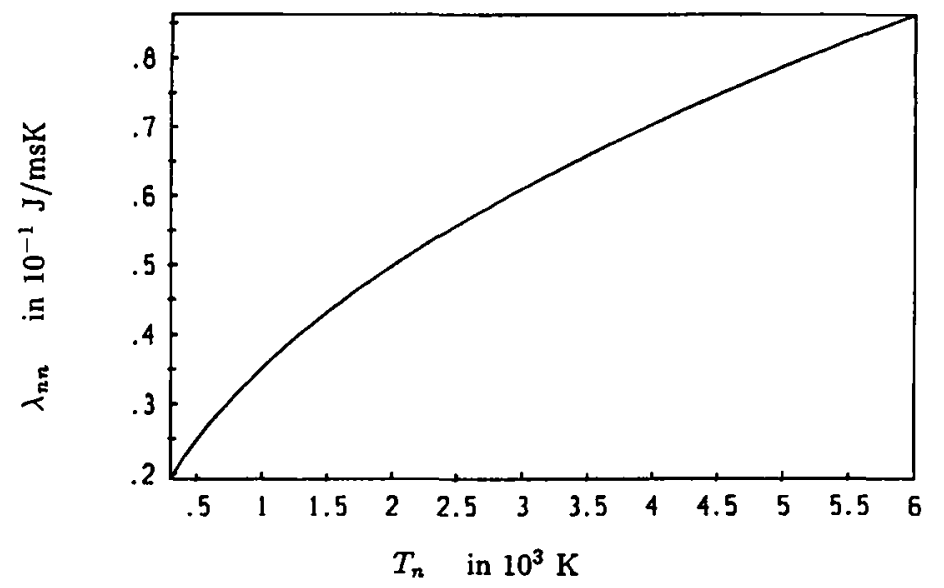

Fig. 13: The thermal conductivity coefficient $\lambda_{n n}$ as a function of the atom temperature $T_{n}$.

\section{Method of Solution}

\subsection{Numerical Problem}

We have to solve a boundary value problem consisting of $n=13$ ordinary differential equations (ODEs)

$$
F_{i}\left(y^{\prime}(x), y(x), x\right)=0 \quad(i=1, \ldots, n)
$$

with $y=\left(y_{1}(x), \ldots, y_{n}(x)\right), y^{\prime}=\mathrm{d} y / \mathrm{d} x$, and we are looking for the solution in the integration domain $0 \leq x \leq R$. We have $r=8$ boundary conditions at $x=0$

$$
A_{i}\left(y^{\prime}(0), y(0)\right)=0 \quad(i=1, \ldots, r)
$$

and $s=4$ boundary conditions at $x=R$

$$
B_{i}\left(y^{\prime}(R), y(R)\right)=0 \quad(i=1, \ldots, s)
$$


and $t=n-r-s=1$ internal conditions at the point $x_{i s}$ inside the integration domain where the ions reach the ion sound velocity

$$
C_{i}\left(y^{\prime}\left(x_{i s}\right), y\left(x_{i s}\right), x_{i s}\right)=0 \quad(i=1, \ldots, t) .
$$

\subsection{Relaxation Method for Boundary Value Problems Involving Critical Points}

We used the code HEMODES, a FORTRAN routine developed by NOBILI and TUROLIA [21]. The code is the implementation of a relaxation method and was explicitly designed to treat systems of ODEs with critical points. In relaxation methods the ODEs are replaced by approximate finite difference equations (FDEs) on a grid or mesh of points that spans the integration domain. We chose $m=1000$ equidistant points

$$
x_{1}=0, x_{2}, \ldots, x_{m}=R \text {. }
$$

At the $m-1$ midpoints of the mesh intervals the dependent variables are interpolated from the values at the mesh points

$$
y\left(x_{i+1 / 2}\right)=\frac{y\left(x_{i+1}\right)+y\left(x_{i}\right)}{2} \quad(i=1, \ldots, m-1),
$$

and their derivatives are approximated by finite differences

$$
y^{\prime}\left(x_{i+1 / 2}\right)=\frac{y\left(x_{i+1}\right)-y\left(x_{i}\right)}{x_{i+1}-x_{i}} \quad(i=1, \ldots, m-1) .
$$

In the $n$ ODEs (97) we substitute the $n$ dependent variables $y_{j}(x)$ by $y_{j}\left(x_{i+1 / 2}\right)$ and their derivatives $y_{j}^{\prime}$ by $y_{j}^{\prime}\left(x_{i+1 / 2}\right)$. Doing this for all $m-1$ half-mesh points we receive a system of $n(m-1)$ difference equations. After the equivalent substitutions in the boundary conditions (98) and (99) and in the internal condition (100) (this can only be done if the location of the critical point $x_{i s}$ was determined in advance) we end up with an algebraic, in general nonlinear system of $m n$ equations in the $m n$ unknowns $z_{k}=z_{i j}=y_{j}\left(x_{i}\right)$

$$
E_{k}\left(z_{1}, \ldots, z_{m n}\right)=0, \quad(k=1, \ldots, m n),
$$

where $E_{k}$ stands for $F_{k}, A_{k}, B_{k}$ or $C_{k}$.

This system is solved using the Newton-Raphson method (Press et al. [30], HÄMmerLin, HOFFMANN [31]). The method reduces the problem of solving a nonlinear system of equations to the problem of iteratively solving a linear system of equations. The iteration procedure requires a trial solution to start with. This trial solution is not required to satisfy the finite difference equations nor the boundary and internal conditions. But with trial solutions too distinct from the true solution the method may not converge. The iteration, here called relaxation, consists of adjusting all the values on the mesh such as to bring them into successively closer agreement with the finite differential equations and simultaneously, with the boundary and internal conditions.

Having a good trial solution $Z^{(0)}$ to start with the function $E_{k}$ is expanded in the surrounding of $Z^{(0)}$ into a Taylor series

$$
E_{k}\left(Z^{(0)}+\delta Z\right)=E_{k}\left(Z^{(0)}\right)+\sum_{l=1}^{m n} \frac{\partial E_{k}}{\partial z_{l}} \delta z_{l}+O\left(\delta Z^{2}\right) \quad(k=1, \ldots, m n) .
$$

If we denote with $\delta z_{l}$ the correction which should be applied to the $l$-th variable in order to improve the trial solution we want the corrected solution $Z^{(0)}+\delta Z$ to satisfy the equations

$$
E_{k}\left(Z^{(0)}+\delta Z\right)=0 \text {. }
$$


Neglecting terms of order $\delta Z^{2}$ and higher, a linear system of equations for the corrections $\delta Z$ has to be solved

$$
\sum_{l=1}^{m n} \frac{\partial E_{k}}{\partial z_{l}} \delta z_{l}=\sum_{l=1}^{m n} g_{k l} \delta z_{l}=-E_{k},(k=1, \ldots, m n)
$$

which can be written in matrix form

$$
\mathbf{G} \cdot \delta Z=-E .
$$

After the solution of this matrix equation the calculated correction $\delta Z$ is added to the trial solution. The iteration process is stopped if the calculated correction is less than the required accuracy.

\subsection{The Special Structure of $\mathbf{G}$ and the Ordering of Dependent Variables}

The $(m n \times m n)$-matrix $\mathbf{G}$ has a special block structure, because using the approximations (101) and (102) for $y$ and $y^{\prime}$ each $g_{k l}$ couples only the mesh points $i$ and $i+1$. Equation (107) is solved for the increments $\delta Z$ using an advanced form of Gaussian elimination which exploits the special block structure of the matrix $\mathbf{G}$ to minimize the storage of matrix coefficients. Only a small subset of the $(m n \times m n)$ matrix elements needs to be stored and manipulated at one time.

This method leads to some limitation on the possible ordering of the dependent variables in the vector

$$
y=\left(y_{1}(x), \ldots, y_{n}(x)\right) \text {. }
$$

This ordering is no more freely choosable. The dependent variables must be stored in a way that the $r$ boundary conditions at the first mesh point $x_{1}$ contain some dependence on the first $r$ dependent variables $y_{1}, \ldots, y_{r}$. Otherwise the square $r \times r$ subsection of the first matrix block treated by the algorithm will appear to be singular. Thus, having boundary conditions at the first mesh point for $Q_{e}, Q_{i}, Q_{n}, V_{e}, V_{i}, V_{n}, \eta_{r}$ and $N_{e}(52-59)$ we stored the dependent variables in the following order:

$$
y=\left(Q_{e}, Q_{i}, Q_{n}, V_{e}, V_{i}, V_{n}, \eta_{n}, N_{e}, N_{i}, N_{n}, \tau_{e}, \tau_{i}, \tau_{n}\right)
$$

\subsection{Trial Solution}

Our trial solution consists of values for the 13 dependent variables $y_{1}(x), \ldots, y_{13}(x)$ on the $m=1000$ mesh points

$$
Z^{(0)}=z_{k}^{(0)}=z_{i j}^{(0)}=y_{i}^{0}\left(x_{j}\right), \quad(i=1, \ldots, 13 ; j=1, \ldots, 1000) .
$$

In order to get a good trial solution to start with we first solved a system of 7 ODEs consisting of the continuity equations, the momentum balance equation and the Poisson equation with constant temperatures and heat currents. As a trial solution for this system we took

$$
\begin{aligned}
& N_{e}\left(x_{j}\right)=1, \\
& N_{i}\left(x_{j}\right)=N_{i 0}, \\
& N_{n}\left(x_{j}\right)=N_{n 0}, \\
& V_{e}\left(x_{j}\right)=V_{e 1} x_{j}, \\
& V_{i}\left(x_{j}\right)=V_{i 1} x_{j},
\end{aligned}
$$




$$
\begin{aligned}
& V_{n}\left(x_{j}\right)=V_{n 1} x_{j}, \\
& \eta_{r}\left(x_{j}\right)=\eta_{r 1} x_{j},
\end{aligned}
$$

where $j=1, \ldots, m$. The boundary value $N_{n 0}$ was calculated with a quasi-neutral model and $N_{i 0}$ was taken from the analytic solution of the simplified equations for a two-fluid model

$$
N_{i 0}=\frac{1}{\lambda} \text { where } \frac{3 N_{n 0}^{2} S_{i o n}^{2}}{2 C} \lambda^{3}+\lambda-1=0
$$

and the $V_{e 1}, V_{i 1}, V_{n 1}$ and $\eta_{r 1}$ are the first coefficients of the Taylor series expansion:

$$
\begin{aligned}
& V_{e 1}=\frac{1}{2} N_{n 0} S_{i o n}, \\
& V_{i 1}=\frac{1}{2} \frac{N_{n 0}}{N_{i 0}} S_{i o n}, \\
& V_{n 1}=-\frac{1}{2} S_{i o n}, \\
& \eta_{r 1}=\frac{1}{2} C\left(N_{i 0}-1\right) .
\end{aligned}
$$

This trial solution turned out not to be close enough to the true solution to ensure convergence of the relaxation method. Therefore, instead of using the constant $C$ in the Poisson equation which is of the order of $10^{5}$ to $10^{6}$ we first solved the equations for a fictitious value $C^{*}$ of the order of 1 to 10 and increased this constant up to its real value in an iterative process. This procedure guaranteed convergence of the solution for the system of $7 \mathrm{ODEs}$. In a second step we took this solution as a trial solution for the system of 13 ODEs with variable temperatures and heat currents. As a trial solution for the 6 new variables we used

$$
\begin{aligned}
\tau_{e}\left(x_{j}\right) & =\tau_{e R}, \\
\tau_{i}\left(x_{j}\right) & =\tau_{i R}, \\
\tau_{n}\left(x_{j}\right) & =\tau_{n R}, \\
Q_{e}\left(x_{j}\right) & =Q_{e 1} x_{j}, \\
Q_{i}\left(x_{j}\right) & =Q_{i 1} x_{j}, \\
Q_{n}\left(x_{j}\right) & =Q_{n 1} x_{j},
\end{aligned}
$$

where the $Q_{e 1}, Q_{11}, Q_{n 1}$ are the first coefficients of the Taylor series expansion for the heat fluxes. Again, we had to ensure convergence by starting with lictitious thermal conductivities of the order of $10^{4}$ and decreasing them in an iterative process down to their real values.

\subsection{Location of Critical Points}

For the set up of the matrix $G$ the exact location of the critical points-in our case the location of the ion sound point $x_{i s}$-has to be known in advance. Therefore, before setting up $\mathrm{G}$ the code HEMODES determines the number and the location of critical points. Critical points occur where the determinant of

$$
\mathrm{U}=\frac{\partial F}{\partial y^{\prime}}
$$


vanishes. HEMODES provides a searching procedure which looks for the interval of the mesh where det $(U(x))$ changes sign. But it can happen that det $(U(x))$ does not change sign even if a critical point is present. This occurs when the function det $(U(x))$ vanishes exactly where it has an extremum. To prevent a failure of the searching procedure a rearrangement of the ODEs by algebraic substitutions is necessary. In our case using equations in the form

$$
\mathrm{U}(y(x), x) y^{\prime}-t(y(x), x)=0
$$

as shown in equations (29) to (41) the searching procedure was successful, while when using the equations in the explicit form

$$
y^{\prime}-U^{-1} \cdot t(x, y(x))=0
$$

no critical point was found.

\section{Results and Discussion}

Using the numerical method described in the section 4 and the material characteristics from section 3 the set of 13 coupled first order differential equations $(20)-(28)$ were solved with the boundary conditions (52) $-(59)$ at the cylinder axis, with the regularity condition (61) and the boundary conditions at the capillary wall $(62)-(65)$. For values which are characteristic for an argon ion laser, the radial dependence of the densities, velocities, temperatures, heat currents, electrical field, pressure and degree of ionization are shown in Figs. 14a-e. In all figures the smooth lines refer to an electron temperature $T_{e R}=50,000 \mathrm{~K}$ at the wall while the dashed lines refer to calculations with $T_{e R}=40,000 \mathrm{~K}$. Furthermore, boundary values $T_{i R}=2,500 \mathrm{~K}$ and $T_{n R}=1,500 \mathrm{~K}$ for the ion and neutral particle temperatures, respectively, have been used throughout as well as an electron density at the axis of $n_{e 0}=2 \times 10^{14} \mathrm{~cm}^{-3}$. The longitudinal electric field $E_{z}$, which can be varied within a certain interval, has been chosen such that the heat current of the electrons at the wall satisfies the condition

$$
q_{e R}=\left(\frac{2}{\pi}-\frac{1}{2}\right) n_{e R} v_{e R} k_{B} T_{e R}
$$

as suggested by VALENTINI [13]. Fig. 14a shows the radial dependences of the electron, ion, and neutral atom densities. The normalized electron and ion densities decrease continuously from 1 on the axis to some finite value at the boundary. Such a behaviour is expected from the Self-Ewald theory. Deviations from quasi-neutrality could practically not be observed within the $r$-region considered and would manifest themselves beyond the Bohm limit in a distance of some Debye lengths (i.e. some $10^{-6} \mathrm{~m}$ ) from the real physical wall. Unfortunately, due to computational difficulties it was not possible to extend the calculations beyond that very small zone; on the other hand, however, one should keep in mind that a hydrodynamic theory with macroscopic transport coefficients looses its sense when applied to changes occurring on these microscopic scales. The neutral density increase with the distance from the axis.

In Fig. $14 \mathrm{~b}$ the velocities of the three components are displayed. While both electron and ion velocity increase strongly with radial distance from the axis until they reach the Bohm velocity, the neutral atom velocity decreases rather slowly. This is due to the fact that no net mass transport occurs in radial direction. Fig. 14c shows the radial variation of the three temperatures. The electron temperature remains almost constant due to the small electron heat conductivity. The ion temperature increases first, reaches a maximum value and decreases near the boundary due to its fixed boundary value. This behaviour is in accordance with kinetic calculations of WALLSCHLÄGER [19] for small Knudsen numbers. The decrease of the neutral gas temperature with increasing $r$ has also been reported in the literature. The $r$-dependence of the three heat flux vectors is displayed in Fig. 14d. While the electron and 

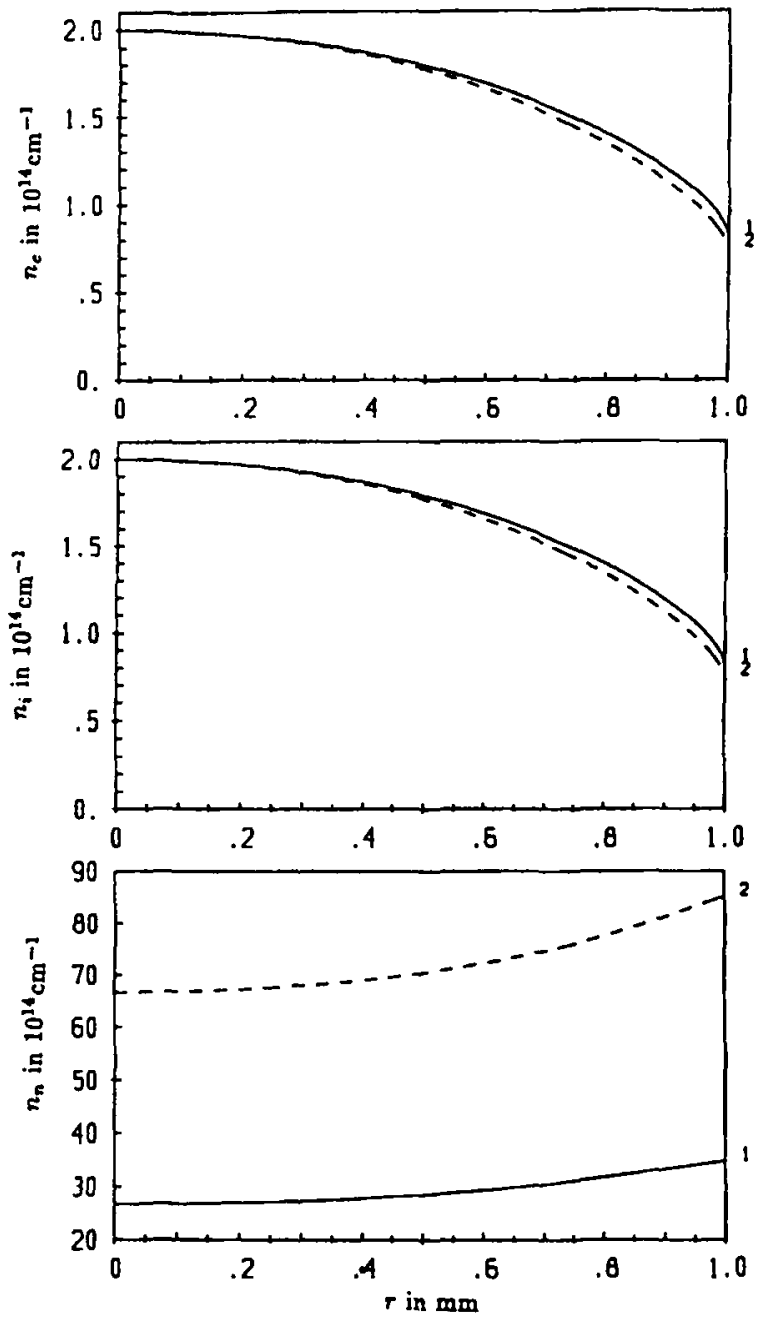

Fig. 14a: Radial dependence of the electron, ion and neutral densities for an electron density on the axis of $2 \times 10^{14} \mathrm{~cm}^{-3}$ and wall temperatures of $2,500 \mathrm{~K}$ for the ions, $1,500 \mathrm{~K}$ for the neutrals and $40,000 \mathrm{~K}$ (dashed line) and $50,000 \mathrm{~K}$ (full line) for the electrons. 

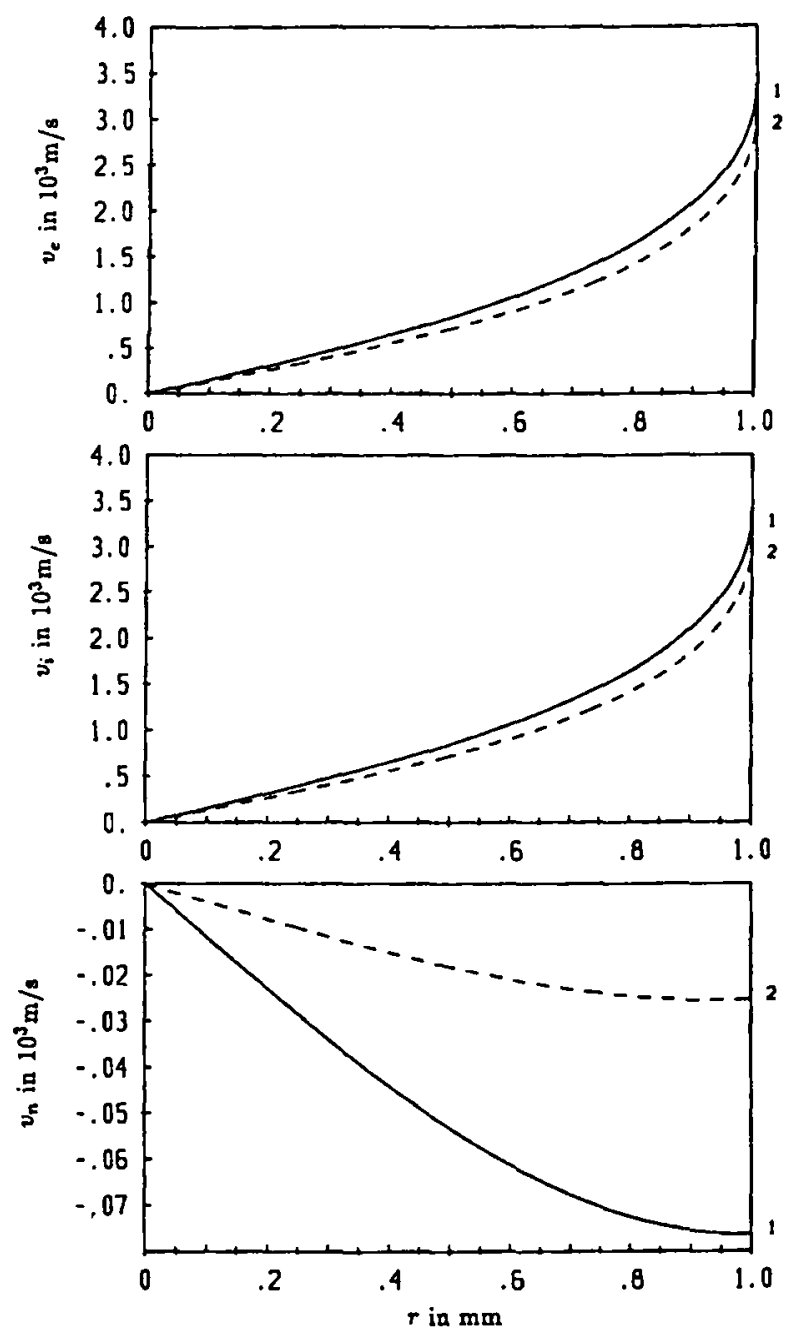

Fig. 14b: Radial dependence of the electron, ion and neutral velocities for an electron density on the axis of $2 \times 10^{14} \mathrm{~cm}^{-3}$ and wall temperatures of $2,500 \mathrm{~K}$ for the ions, $1,500 \mathrm{~K}$ for the neutrals and $40,000 \mathrm{~K}$ (dashed line) and $50,000 \mathrm{~K}$ (full line) for the electrons. 

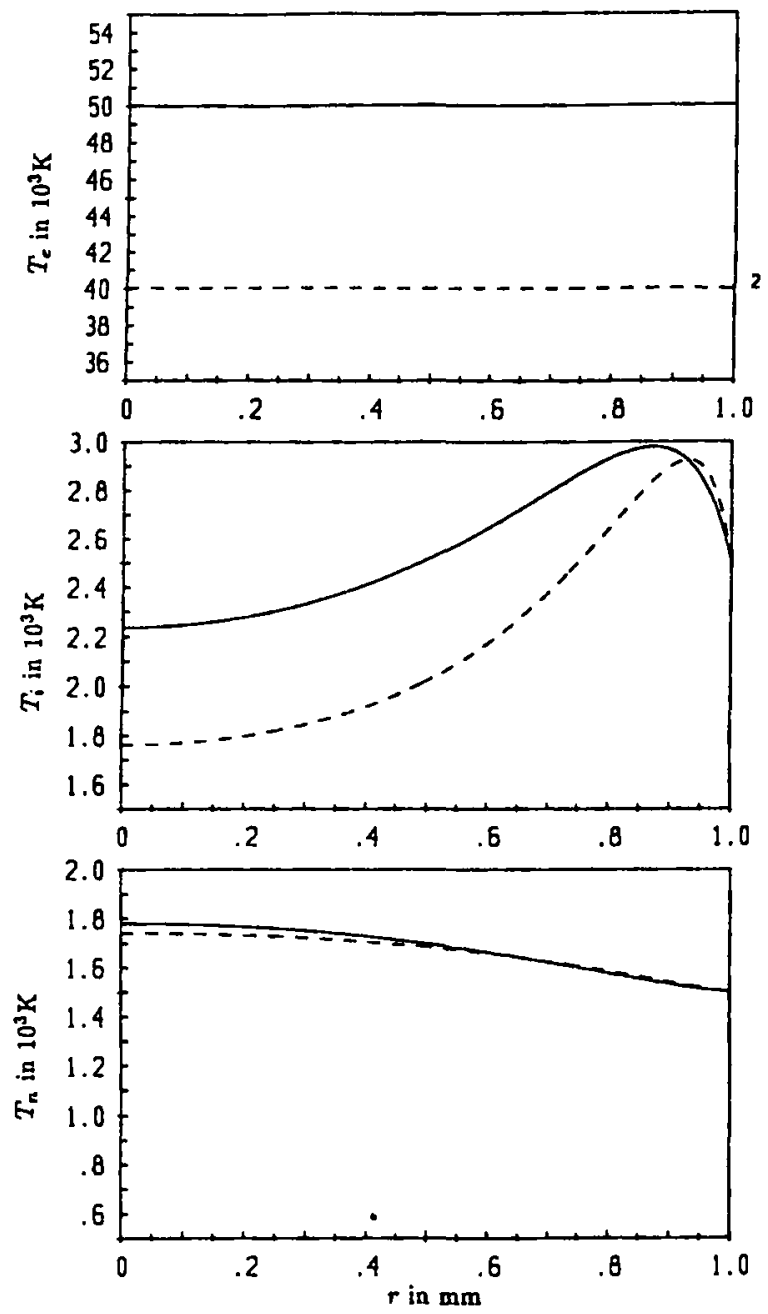

Fig. 14c: Radial dependence of the electron, ion and neutral temperatures for an electron density on the axis of $2 \times 10^{14} \mathrm{~cm}^{-3}$ and wall temperatures of $2,500 \mathrm{~K}$ for the ions, $1,500 \mathrm{~K}$ for the neutrals and $40,000 \mathrm{~K}$ (dashed line) and $50,000 \mathrm{~K}$ (full line) for the electrons. 

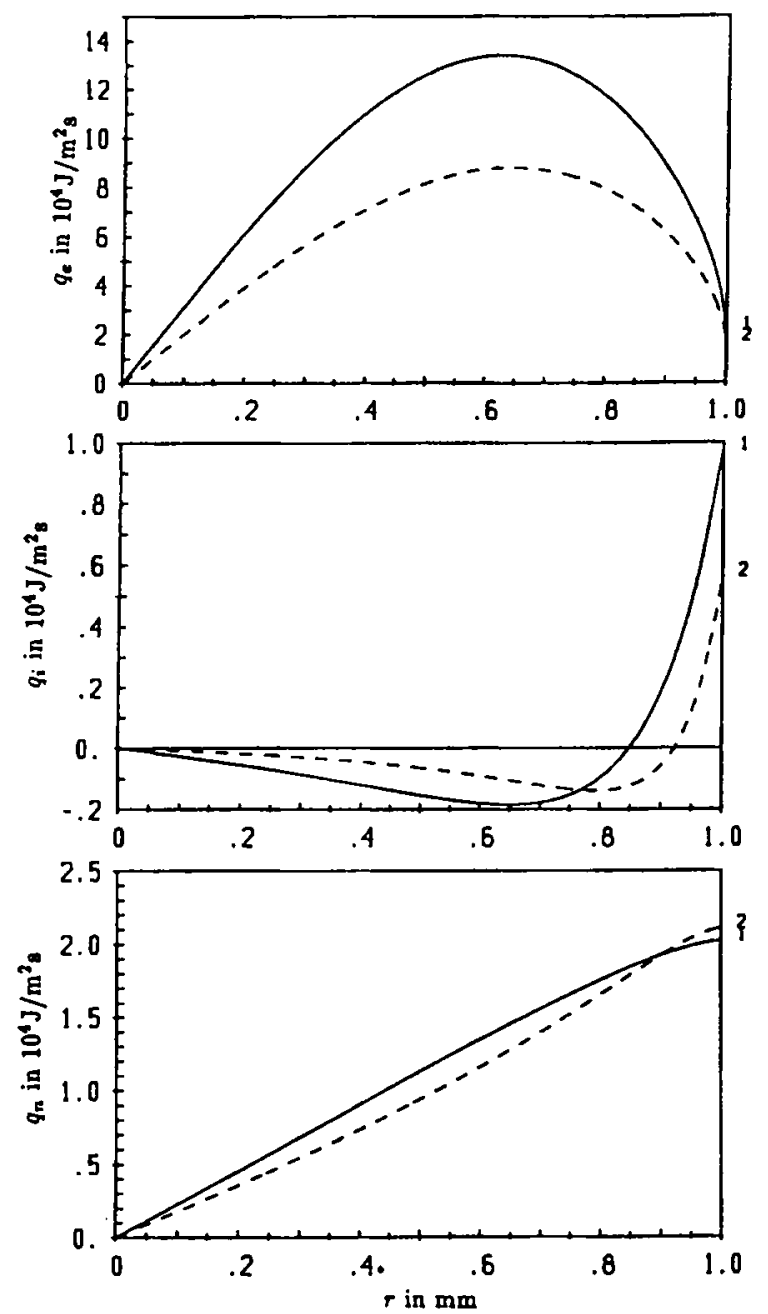

Fig. 14d: Radial dependence of the electron, ion and neutral heat currents for an electron density on the axis of $2 \times 10^{14} \mathrm{~cm}^{-3}$ and wall temperatures of $2,500 \mathrm{~K}$ for the ions, $1,500 \mathrm{~K}$ for the neutrals and $40,000 \mathrm{~K}$ (dashed line) and $50,000 \mathrm{~K}$ (full line) for the electrons. 

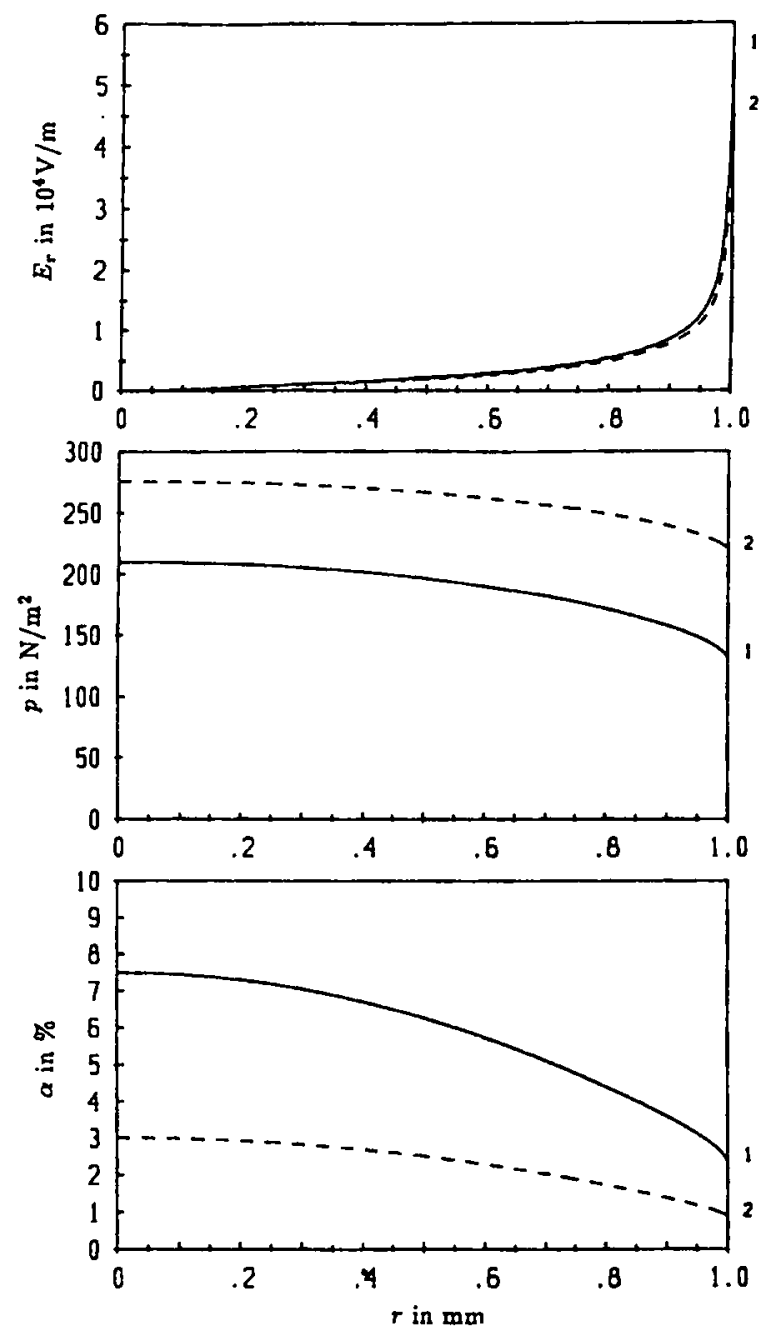

Fig. 14e: Radial dependence of the radial component of the electrical field, total pressure and degree of ionization for an electron density on the axis of $2 \times 10^{14} \mathrm{~cm}^{-3}$ and wall temperatures of $2,500 \mathrm{~K}$ for the ions, $1,500 \mathrm{~K}$ for the neutrals and $40,000 \mathrm{~K}$ (dashed line) and $50,000 \mathrm{~K}$ (full line) for the electrons. 
neutral gas heat fluxes remain positive, the ionic heat flux changes sign. The negative ionic heat flux is caused by the initial increase of the ion temperature. Finally, Fig. 14.e shows the radial dependence of the radial electric field, the total pressure

$$
p=n_{e} k_{B} T_{e}+n_{i} k_{B} T_{i}+n_{n} k_{B} T_{n},
$$

and the degree of ionization $\alpha \approx n_{i} / n_{n}$. The electric field shows a steep increase near the wall due to deviations from quasi-neutrality. The correct field value at the wall, however, cannot be reproduced with a hydrodynamic approach as already mentioned and thus, the measured wall potential which is roughly given by $5 k_{B} T_{e}$ for argon is not quite reached. While the total pressure decreases slightly, the decrease of the ionization coefficient is more pronounced, in particular for the higher boundary value of the electron temperature.

The code can be used to investigate the effect of changing various parameters on the radial dependence of physical variables and on measurable quantities like the radially averaged degree of ionization and the average total pressure. As an example, in Table 1 (for an electron temperature of $50,000 \mathrm{~K}$ ) and in Table 2 (for an electron temperature of $60,000 \mathrm{~K}$ ) the variation of the average degree of ionization, the electrical current, the average total gas pressure, the longitudinal electrical field and the average neutral particle density are presented for electron densities on the axis ranging from $3 \times 10^{13}-2 \times 10^{14} \mathrm{~cm}^{-3}$ and for an ion and a neutral gas temperature of $2,000 \mathrm{~K}$.

Tab. 1:

Radially averaged degree of ionization, electric current, average total pressure, longitudinal electric field and average neutral gas density for an electron temperature of $50,000 \mathrm{~K}$ and an ion and a neutral gas temperature of $2,000 \mathrm{~K}$.

\begin{tabular}{cccccc}
\hline$n_{e 0}\left[\mathrm{~m}^{-3}\right]$ & $\bar{\alpha}[\%]$ & $I[\mathrm{~A}]$ & $\bar{p}\left[\mathrm{~N} / \mathrm{m}^{2}\right]$ & $E_{z}[\mathrm{~V} / \mathrm{m}]$ & $\bar{n}_{n}\left[\mathrm{~m}^{-3}\right]$ \\
\hline $3 \cdot 10^{19}$ & 0.733 & 2.024 & 99 & 574.1 & $3.030 \cdot 10^{21}$ \\
$4 \cdot 10^{19}$ & 0.980 & 2.527 & 104 & 592.4 & $3.028 \cdot 10^{21}$ \\
$5 \cdot 10^{19}$ & 1.227 & 2.998 & 110 & 607.7 & $3.027 \cdot 10^{21}$ \\
$6 \cdot 10^{19}$ & 1.475 & 3.444 & 115 & 621.2 & $3.026 \cdot 10^{21}$ \\
$7 \cdot 10^{19}$ & 1.723 & 3.871 & 121 & 633.1 & $3.025 \cdot 10^{21}$ \\
$8 \cdot 10^{19}$ & 1.972 & 4.282 & 126 & 644.0 & $3.024 \cdot 10^{21}$ \\
$9 \cdot 10^{19}$ & 2.221 & 4.678 & 131 & 654.0 & $3.023 \cdot 10^{21}$ \\
$1.0 \cdot 10^{20}$ & 2.470 & 5.062 & 136 & 663.0 & $3.023 \cdot 10^{21}$ \\
$1.1 \cdot 10^{20}$ & 2.720 & 5.436 & 142 & 672.1 & $3.022 \cdot 10^{21}$ \\
$1.2 \cdot 10^{20}$ & 2.971 & 5.799 & 148 & 680.4 & $3.022 \cdot 10^{21}$ \\
$1.3 \cdot 10^{20}$ & 3.221 & 6.155 & 152 & 688.2 & $3.022 \cdot 10^{21}$ \\
$1.4 \cdot 10^{20}$ & 3.472 & 6.502 & 158 & 695.7 & $3.021 \cdot 10^{21}$ \\
$1.5 \cdot 10^{20}$ & 3.724 & 6.842 & 163 & 702.8 & $3.021 \cdot 10^{21}$ \\
$1.6 \cdot 10^{20}$ & 3.976 & 7.176 & 169 & 709.7 & $3.021 \cdot 10^{21}$ \\
$1.8 \cdot 10^{20}$ & 4.480 & 7.825 & 180 & 722.9 & $3.020 \cdot 10^{21}$ \\
$2.0 \cdot 10^{20}$ & 4.985 & 8.455 & 191 & 735.2 & $3.020 \cdot 10^{21}$
\end{tabular}


Tab. 2:

Radially averaged degree of ionization, electric current, average total pressure, longitudinal electric field and average neutral gas density for an electron temperature of $60,000 \mathrm{~K}$ and an ion and a neutral gas temperature of $2,000 \mathrm{~K}$.

\begin{tabular}{cccccc}
\hline$n_{\mathrm{e} 0}\left[\mathrm{~m}^{-3}\right]$ & $\bar{\alpha}[\%]$ & $I[\mathrm{~A}]$ & $\bar{p}\left[\mathrm{~N} / \mathrm{m}^{2}\right]$ & $E_{z}[\mathrm{~V} / \mathrm{m}]$ & $\bar{n}_{n}\left[\mathrm{~m}^{-3}\right]$ \\
\hline $5 \cdot 10^{19}$ & 2.303 & 3.911 & 76.79 & 532.8 & $1.624 \cdot 10^{21}$ \\
$6 \cdot 10^{19}$ & 2.768 & 4.495 & 83.18 & 544.5 & $1.623 \cdot 10^{21}$ \\
$7 \cdot 10^{19}$ & 3.233 & 5.056 & 89.58 & 555.2 & $1.622 \cdot 10^{21}$ \\
$8 \cdot 10^{19}$ & 3.698 & 5.599 & 95.99 & 565.3 & $1.622 \cdot 10^{21}$ \\
$9 \cdot 10^{19}$ & 4.163 & 6.127 & 102.39 & 574.8 & $1.621 \cdot 10^{21}$ \\
$1.0 \cdot 10^{20}$ & 4.628 & 6.643 & 108.80 & 584.0 & $1.621 \cdot 10^{21}$ \\
$1.1 \cdot 10^{20}$ & 5.093 & 7.150 & 115.19 & 592.9 & $1.620 \cdot 10^{21}$ \\
$1.2 \cdot 10^{20}$ & 5.557 & 7.648 & 121.59 & 601.6 & $1.620 \cdot 10^{21}$ \\
$1.3 \cdot 10^{20}$ & 6.020 & 8.141 & 127.97 & 610.0 & $1.619 \cdot 10^{21}$ \\
$1.4 \cdot 10^{20}$ & 6.483 & 8.628 & 134.35 & 618.5 & $1.619 \cdot 10^{21}$ \\
$1.5 \cdot 10^{20}$ & 6.945 & 9.113 & 140.71 & 626.8 & $1.619 \cdot 10^{21}$ \\
$1.6 \cdot 10^{20}$ & 7.405 & 9.595 & 147.06 & 635.1 & $1.618 \cdot 10^{21}$ \\
$1.7 \cdot 10^{20}$ & 7.865 & 10.075 & 153.39 & 643.4 & $1.618 \cdot 10^{21}$ \\
$1.8 \cdot 10^{20}$ & 8.325 & 10.556 & 159.71 & 651.7 & $1.617 \cdot 10^{21}$ \\
$1.9 \cdot 10^{20}$ & 8.779 & 11.038 & 166.00 & 660.0 & $1.617 \cdot 10^{21}$ \\
$2.0 \cdot 10^{20}$ & 9.234 & 11.521 & 172.28 & 668.4 & $1.616 \cdot 10^{21}$
\end{tabular}

\section{Conclusion}

It has been demonstrated that a simulation of the radial dependences of plasma parameters in the positive column of an argon discharge with relatively large Knudsen number, based on local balance and transport equations, can successfully be performed up to distances of a few Debye lengths from the physical wall. Thereby, the equations pertaining to a three-fluidmodel supplemented with appropriate boundary conditions have been solved by means of the boundary value code HEMODES which, in addition, correctly handles internal singular points, e.g. the ion sound point. Although, this approach is a hydrodynamical one, Boltzmann kinetic theory is involved in so far that it has to provide expressions for the relevant transport coefficients. In order to describe the (very thin) plasma-wall-sheath correctly, however, a full kinetic treatment seems to be inevitable. The present calculations represent a first step for the simulation of the argon ion laser plasma. For a comparison of the results with experimentally accessible quantities (e.g. the longitudinal electrical field strength), however, it might be necessary to allow also for ionization from excited atomic states. This will enlarge the system of equations to be solved considerably and will be subject of a future investigation.

This work was sponsored in part by the German Ministry of Research and Technology (BMFT).

\section{References}

[1] Tonks, L., Langmuir, I., Phys. Rev. 34 (1929) 876.

[2] Sснотtкy, W., Physik. Z. 25 (1924) 635.

[3] Persson, K. B., Phys. Fluids 9 (1962) 1625.

[4] Self, S. A., Ewald, H. N., Phys. Fluids 9 (1966) 2486.

[5] Forrest, J. R., Franklin, R. N., Brit. J. Appl. Phys. 17 (1966) 1061

[6] Forrest, J. R.. Franklin, R. N., J. Phys. D: Appi. Phys. 1 (1968) 1357.

[7] Bонм, D., in "The characteristics of electrical discharges in magnetic fields", eds. A. Guthrie and R. K. Wakerling, McGraw-Hill, New York (1949). 
[8] Kino, G. S., Shaw, E. K., Phys. Fluids 9 (1966) 587.

[9] Valentini, H.-B., Beitr. Plasmaphys. 19 (1979) 151.

[10] Friedman, H. W., Levi, E., Phys. Fluids 10 (1967) 1499.

[11] INGOLd, J. H., Phys. Fluids 15 (1972) 75.

[12] Valentini, H.-B., J. Phys. D: Appl. Phys. 21 (1988) 311.

[13] Valentini, H.-B., Contrib. Plasma Phys. 31 (1991) 211.

[14] Metze, A., Ernie, D. W., Oskam, H. J., Phys. Rev. A 39 (1989) 4117.

[15] Frankin, R. N., "Plasma Phenomena in Gas Discharges", Clarendon Press, Oxford (1976).

[16] Valentini, H.-B., Contrib. Plasma Phys. 31 (1991) 391.

[17] ILIC, D. B., J. Appl. Phys. 44 (1973) 3993.

[18] Valentini, H.-B., Beitr. Plasmaphys. 20 (1980) 243.

[19] Wallschläger, H., Contrib. Plasma Phys. 30 (1990) 385.

[20] Henyey, L. G., Forbes J. E., Gould, N. L., Astrophys. J. 139 (1964) 306.

[21] Nobili, L., Turolla, R., Astrophys. J. 333 (1988) 248.

[22] TIEMANN, W., Verh. DPG (VI) 24 (1989) 41.

[23] SCHWAB, C., KöhLER, W. E., Verh. DPG (VI) 24 (1989) 42.

[24] Mitchner, M., Kruger, C. H., "Partially Ionized Gases", Wiley and Sons, New York (1973).

[25] StOER, J., BulirsCh, R., "Numerische Mathematik 2", Springer-Verlag, Berlin (1990).

[26] Stephan, K., Helm, H., MärK, T., J. Chem. Phys. 73 (1980) 3763.

[27] KRENZ, J., “Daten zur Reaktionskinetik von ArF-Lasern”, BMFT-Projekt Nr. 13N54069 (Interner Bericht), Institut für Plasmaphysik, Universität Hannover (1987).

[28] Chapman, S., Cowling, T. G., "Mathematical Theory of nonuniform gases", Cambridge University Press, Cambridge (1939).

[29] McCourt, F., Beenakker, J., Köhler, W., Kusčer, I., "Nonequilibrium Phenomena in Polyatomic Gases, Volume I, Dilute Gases", Clarendon Press, Oxford (1990).

[30] Press, W., Flannery, B. P., Teukolsky, S., Vetrerling, W. T., "Numerical Recipes. The Art of Scientific Computing", Cambridge University Press, Cambridge (1986).

[31] Hämmerdin, G., Hoffmann, K.-H., "Numerische Mathematik", Springer-Verlag, Berlin (1989).

Received March 31, 1992;

revised manuscript received May 1I, 1992 